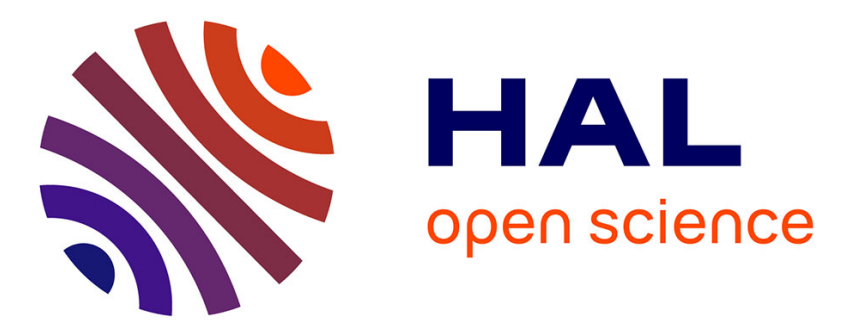

\title{
L'ajustement microéconomique des prix des carburants en France
}

Erwan Gautier, Ronan Le Saout

\section{To cite this version:}

Erwan Gautier, Ronan Le Saout. L'ajustement microéconomique des prix des carburants en France. 2015. hal-01195759

\author{
HAL Id: hal-01195759 \\ https://hal.science/hal-01195759
}

Preprint submitted on 8 Sep 2015

HAL is a multi-disciplinary open access archive for the deposit and dissemination of scientific research documents, whether they are published or not. The documents may come from teaching and research institutions in France or abroad, or from public or private research centers.
L'archive ouverte pluridisciplinaire HAL, est destinée au dépôt et à la diffusion de documents scientifiques de niveau recherche, publiés ou non, émanant des établissements d'enseignement et de recherche français ou étrangers, des laboratoires publics ou privés. 
EA 4272

\title{
L'ajustement microéconomique des prix des carburants en France
}

\author{
Erwan Gautier* ** \\ Ronan Le Saout ${ }^{\star \star *}$
}

$2015 / 22$

$\left(^{*}\right)$ LEMNA-TEPP, Université de Nantes

$\left({ }^{* *}\right)$ Banque de France

$\left.{ }^{(* *}\right)$ CREST et Ecole Polytechnique

Laboratoire d'Economie et de Management Nantes-Atlantique Université de Nantes

Chemin de la Censive du Tertre - BP 52231

44322 Nantes cedex 3 - France

www.univ-nantes.frliemn-iae/recherche

Tél. +33 (0)2 40141717 - Fax +33 (0)2 40141749 


\section{L'ajustement microéconomique des prix des carburants en France}

Erwan GAUTIER (LEMNA-TEPP, Université de Nantes et Banque de France. Email: erwan.gautier@univ-nantes.fr)

Ronan LE SAOUT (CREST et Ecole Polytechnique)

Nous tenons à remercier L. Baudry et G. Retout pour leur précieuse assistance de recherche et E. Chamayou pour ces données sur les services dans les stations-service. Nous remercions deux rapporteurs anonymes de la revue ainsi que les participants aux différents séminaires et conférences au cours desquels le papier a été présenté pour leurs suggestions et commentaires. E. Gautier remercie la chaire «Finance » de la Fondation de projets de l'Université de Nantes pour son soutien financier. Les idées exposées dans cette étude ne reflètent pas nécessairement l'opinion de la Banque de France. 


\section{Résumé long}

Les variations des prix des carburants sont fréquentes et d'ampleur souvent importante, ce qui suscite, parmi les consommateurs, des inquiétudes et des incertitudes sur leur pouvoir d'achat. C'est aussi la raison pour laquelle les prix des carburants sont suivis avec attention par les pouvoirs publics comme en témoignent les nombreux rapports rendus sur le sujet (Bellec et al. (2012) pour un exemple récent). Comment sont modifiés les prix des carburants en France et à quelle vitesse s'ajustent-ils ? Cet article propose de décrire au niveau des stations-service, les modes d'ajustements des prix des carburants et leurs déterminants.

Depuis 2005, de nombreux travaux empiriques ont mis en évidence que les prix à la consommation n'étaient pas modifiés en continu mais plutôt de façon irrégulière. Les prix sont alors considérés comme rigides s'ils réagissent avec retard aux chocs économiques. Une des difficultés est d'observer la fréquence et la nature des chocs affectant le coût à produire au niveau microéconomique. Les prix des carburants apparaissent comme un cas intéressant à étudier car on peut approcher une grande partie de ces coûts en utilisant les prix de gros du produit fini cotés sur le marché européen. Cet avantage va nous permettre de mieux comprendre pourquoi les prix changent de façon irrégulière et de relier nos observations aux théories existantes justifiant la rigidité des prix.

Généralement, les variations des prix des carburants sont expliquées à un niveau agrégé à l'aide de données macroéconomiques. Nous proposons dans cet article d'utiliser des données microéconomiques de prix relevés dans les stations-services à une fréquence quotidienne. Ces prix sont issus du site internet gouvernemental http://www.prixcarburants.gouv.fr sur une période allant de janvier 2007 à juin 2009 et couvrent plus des trois quarts des stations-service en France. Cette approche microéconomique nous permet d'analyser l'hétérogénéité des comportements de fixation des prix des carburants, notamment le lien entre concurrence et rigidité des prix. Pour cela, nous étudions au niveau de chaque station-service, les durées entre deux changements de prix et les tailles de ces changements, puis nous relions ces variables aux différentes caractéristiques des stations-services (type de distributeur, concurrence locale par exemple). Enfin, nous analysons comment les stationsservice transmettent à leur prix des variations du prix de gros des carburants sur le marché international.

En France, les prix des carburants ne sont pas modifiés quotidiennement mais en moyenne une fois par semaine. Les stations-service des supermarchés changent leur prix plus fréquemment que les autres distributeurs. Toutes choses égales par ailleurs, plus la concurrence locale est forte, plus les prix sont modifiés fréquemment. Les prix dits psychologiques, se terminant par un « $0 »$ ou un « $9 »$, ont des durées plus longues. En moyenne, la taille de changements de prix hors taxe est comprise entre 2,5 et $3 \%$ à la hausse comme à la baisse et les changements de prix inférieurs à $2 \%$ sont rares. Enfin, il existe une corrélation positive entre taille des changements et durée des prix entre deux changements. $\mathrm{Au}$ niveau agrégé, une augmentation des prix des carburants s'explique principalement par une hausse de la fréquence des changements de prix et non par une hausse de la taille moyenne des changements. Certains jours de la semaine sont plus propices aux changements de prix notamment le mardi et le vendredi alors que peu de changements sont observés entre le samedi et le lundi. En outre, les variations de la fréquence des changements de prix sont corrélées aux variations des cours internationaux des produits raffinés. Le délai de réaction des stations-service aux variations des cours internationaux est de l'ordre de deux semaines. 
Théoriquement, les modèles associant coût à l'ajustement des prix et information imparfaite apparaissent comme les plus prometteurs pour reproduire les faits stylisés mis en évidence dans cette étude.

Cette étude présente des faits stylisés sur la fixation des prix des carburants et met en évidence de façon indirecte la présence de rigidités de prix. Une piste de recherche ultérieure pourrait consister à mesurer et tester directement la nature et l'ampleur des coûts d'ajustement des prix dans les stations-service via par exemple des enquêtes. 


\section{Introduction}

Au cours des années 2000, deux caractéristiques importantes de l'évolution du prix du pétrole apparaissent : le prix du Brent a plus que doublé, et sa variabilité s'est accrue. Ces mouvements ont suscité des inquiétudes sur ses conséquences pour l'activité économique et le pouvoir d'achat des ménages. En particulier, la volatilité des prix des carburants a fortement augmenté. Cet article propose d'étudier avec des données individuelles les modes d'ajustement des prix des carburants distribués en France.

Quelle est la dynamique des prix des carburants ? Une première contribution de cette étude est d'apporter de nouveaux faits stylisés microéconomiques sur l'ajustement des prix des carburants en France ${ }^{i}$. Ces dernières années, une littérature empirique abondante a eu pour objectif de décrire les modes d'ajustement des prix à la consommation et leurs conséquences sur l'inflation. Pour cela, les données mensuelles de relevés de prix utilisés pour construire l'indice de prix à la consommation sont analysées en détailii. Toutefois, pour les carburants, ces relevés mensuels ne permettent pas de rendre compte précisément d'ajustements de prix à fréquence plus élevée. Dans cette étude, nous documentons de nouveaux faits sur la fréquence et la distribution des changements de prix des carburants à partir d'une base de relevés individuels quotidiens. L'étude spécifique de la rigidité des prix des carburants est intéressante pour au moins deux raisons. Tout d'abord, les études disponibles sur la rigidité des prix des carburants portent sur les États-Unis et sur un nombre limité de stations (Davis et Hamilton, 2004 ; Davis, 2007 ; Hosken et al., 2008). Dans cette étude, nous utilisons une base de données originale constituée de relevés quotidiens de prix des carburants pour un peu plus de 10000 stations-service en France sur la période janvier 2007 - juin 2009. La base de données contient au total environ 8,5 millions relevés de prix, ce qui nous permet d'obtenir des indicateurs de rigidité des prix représentatifs de l'ensemble du marché français de la distribution de carburants. La deuxième raison justifiant l'analyse spécifique des prix des carburants est qu'elle nous permet de caractériser l'effet de la concurrence sur les modes d'ajustement des prix. Rotemberg et Saloner (1987) montrent théoriquement que les prix sont moins rigides quand la concurrence est forte mais les résultats empiriques sont relativement peu nombreux (Bils et Klenow, 2004). Notre base de données couvre la presque totalité des distributeurs de carburants et contient des informations sur leur localisation. En outre, les carburants peuvent être considérés comme des biens homogènes dont la qualité et la composition varient peu entre distributeurs. Ceci nous permet de mesurer précisément le lien entre fréquence et taille des changements de prix et concurrence locale. Nous apportons ainsi des résultats empiriques nouveaux sur ce lien.

Une deuxième contribution de cet article est de relier les caractéristiques d'ajustement des prix aux prédictions des modèles théoriques de rigidité des prix utilisés dans les modèles macroéconomiques. En effet, plusieurs modèles justifient souvent l'existence de rigidités nominales de prix. Cependant, leurs conséquences pour les effets de la politique monétaire peuvent différer (Nakamura et Steinsson, 2013). Il apparaît essentiel de pouvoir valider ou invalider empiriquement les prédictions de ces différents modèles. Une des difficultés de la littérature empirique sur la rigidité des prix est de relier la fréquence des changements de prix au concept théorique de la rigidité des prix (Kempf, 2006). En effet, si les chocs de coût subis par un produit sont peu fréquents, le prix sera modifié rarement sans qu'il soit rigide. La faible fréquence des changements pourrait pourtant suggérer à tort que le prix de ce produit est rigide. Un des déterminants importants des prix des carburants est le prix du pétrole qui connaît des variations quotidiennes de large ampleur, ce qui devrait impliquer des changements de prix eux aussi quotidiens. La fréquence des changements de prix ou la durée 
de fixité des prix est alors un bon indicateur de rigidité des prix. L'étude des prix des carburants permet donc d'obtenir des éléments complémentaires aux analyses portant sur l'ensemble des prix à la consommation. Par ailleurs, les résultats empiriques sur la rigidité des prix sont souvent obtenus dans des environnements économiques relativement stables où la taille des chocs est faible. La fréquence et la taille des chocs sur le prix du pétrole sur la période 2007-2009 nous permettent de mieux identifier les caractéristiques de l'ajustement des prix quand les chocs sont de grande taille $\mathrm{e}^{\mathrm{iii}}$. Ainsi, la plupart des études montrent que la fréquence des changements de prix est stable dans le temps, ce qui suggère que la décision de changement de prix est indépendante du contexte économique. Dans cette étude, nous relions les variations temporelles de la fréquence et de la taille des changements de prix à l'inflation agrégée des prix des carburants et aux variations du prix du pétrole raffiné. Notre objectif est double : tester les prédictions des modèles théoriques en présence de chocs fréquents et de grande taille, et évaluer l'effet des chocs sur le prix du pétrole raffiné sur la fréquence et la taille des changements de prix.

L'organisation de l'article est la suivante. La première partie présente les données individuelles de prix utilisées. La deuxième partie donne les principaux résultats sur la fréquence des changements de prix des carburants alors que la troisième partie se concentre sur la distribution des changements de prix. Ces deux parties mettent en évidence quelques déterminants de l'hétérogénéité des modes d'ajustement entre stations-service. Dans la dernière partie, nous étudions les contributions aux variations temporelles de l'inflation des prix des carburants de la marge extensive - la fréquence des changements - et de la marge intensive - la taille moyenne des changements (en excluant les variations de prix nulles) - et leurs déterminants.

\section{Données microéconomiques sur les prix des carburants en France}

Notre base de données contient les prix individuels de toutes les stations-service vendant plus de $500 \mathrm{~m}^{3}$ de carburants par an en France. Depuis janvier 2007, les stationsservice ont l'obligation de signaler au Ministère de l'Economie tous leurs changements de prix pour l'essence sans plomb 95 (SP 95) et le diesel. Les données collectées sont ensuite rendues publiques sur un site internet gouvernemental (http://www.prix-carburants.gouv.fr). Ce site vise à favoriser la concurrence en fournissant une information publique et gratuite sur les prix. D'autres sites internet privés proposent des services similaires mais la mise à jour des prix est alors volontaire. Dans notre cas, l'administration publique peut contraindre les distributeurs à signaler leurs changements de prix ${ }^{\mathrm{i} v}$.

Les principales variables disponibles dans notre base de données sont les suivantes : (i) le prix de vente d'un litre de diesel et de SP 95 (en euros), ce prix inclut les taxes, exprimé en euros avec trois décimales; (ii) la date de relevé du prix sous le format JJ/MM/AA, ce qui permet de suivre l'évolution des prix pour une station-service donnée; (iii) un numéro d'identification de chaque distributeur. Par ailleurs, quelques informations sur le détaillant sont disponibles : marque, localisation et services proposés par la station-service.

Notre base de données est constituée de l'historique des prix extraits chaque jour à 23 h59 à partir du site gouvernemental du $1^{\text {er }}$ janvier 2007 au 31 mai 2009; au total, elle contient plus de 10000 stations-service vendant du diesel et du SP 95 et environ 8,5 millions relevés de prix par carburants. Le marché français des carburants est constitué de quatre types de distributeurs : (i) les stations-service associées aux supermarchés ; (ii) les stations-service appartenant à des groupes pétroliers tels que Total, Elf, Shell, etc. ; (iii) les petits distributeurs 
indépendants sans relation contractuelle avec un groupe pétrolier et vendant uniquement des carburants; (iv) les stations-service situées sur autoroute qui appartiennent souvent à des groupes pétroliers. Une caractéristique importante est que le niveau des prix est en moyenne plus faible dans les supermarchés que dans les autres stations-service. Les supermarchés représentent $60 \%$ des stations-service en termes de parts de marché en 2007 mais seulement $40 \%$ en nombre de stations-service. Notre base de données inclut environ 4500 stationsservice associées à des supermarchés et environ 5500 pour les autres types de distributeurs.

Le prix des carburants peut être décomposé en trois principaux éléments : (i) le prix d'achat du pétrole (après raffinage) qui représente $75 \%$ à $85 \%$ des dépenses totales d'exploitation ${ }^{v}$; (ii) les coûts de distribution, qui incluent les coûts du travail et de transport, et ne sont pas observés dans notre étude. Nous pouvons toutefois supposer qu'ils ne changent pas à une fréquence quotidienne ; (iii) les taxes. En 2009, les deux principales taxes, la Taxe sur la valeur ajoutée (TVA) et la Taxe intérieure sur les produits pétroliers (TIPP), représentent $65 \%$ du prix du SP 95 et $60 \%$ du prix du diesel. La TIPP est assise sur les volumes et non sur le prix de vente des carburants; elle peut être révisée en janvier chaque année mais est restée fixe entre 2007 et $2009^{\text {vi }}$. Le taux de TVA est de 19,6\% (13\% en Corse), et n'a pas subi de modification sur notre période d'étude. La TVA est assise sur le prix incluant la TIPP. Nous calculons le prix hors taxes (HT) $p_{H T}$ de la façon suivante : $p_{H T}=\frac{p_{T T C}}{1+T V A}-T I P P$ où $p_{T T C}$ le prix incluant toutes les taxes, TVA est le taux de TVA et TIPP le montant de la TIPP par litre. Le prix HT moyen d'un litre de diesel varie de 36 à 80 centimes d'euros pour une moyenne de 54 centimes sur notre période d'étude (Graphique 1). Le prix HT d'un litre de SP 95 varie de 29 à 66 centimes d'euros pour une moyenne de 49 centimes. La fiscalité des carburants joue un rôle d'amortisseur des chocs sur les prix TTC. Une augmentation d'un centime du prix HT (soit $2 \%$ pour un prix de 50 centimes) se traduira par une augmentation d'environ $1,1 \%$ du prix TTC pour le diesel et de $0,9 \%$ pour le SP 95 . Cependant, une hausse du prix HT aura des conséquences légèrement différentes sur le prix TTC selon le niveau du prix HT. Par exemple, quand le prix HT moyen du diesel est de 36 centimes, une hausse de $2 \%$ sur le prix HT se traduira par une hausse de $0,9 \%$ sur le prix TTC alors que quand le prix HT est de 80 centimes, cette même hausse du prix HT se traduira par une hausse légèrement plus importante sur le prix TTC (1,3\%). Dans cette étude, nous analysons le comportement de fixation des prix de l'entreprise qui est implicitement le résultat d'une maximisation de ses profits calculés à partir des prix HT, c'est pourquoi nous concentrons notre analyse sur l'étude des prix HT. Toutefois, la réaction des consommateurs peut influer ce comportement : après une hausse de prix TTC, la demande peut diminuer ${ }^{\text {vii }}$. Rotemberg (2005) montre ainsi théoriquement que la rigidité des prix peut s'expliquer par la réaction hostile des consommateurs aux hausses de prix. Nous présentons donc dans cette étude des résultats sur la taille des changements de prix pour les prix HT et TTC et leurs différences. 
Graphique 1 : Prix moyens des carburants calculés à partir des relevés individuels, prix des carburants publiés par le Ministère de l'Economie et prix de marché à Rotterdam des carburants
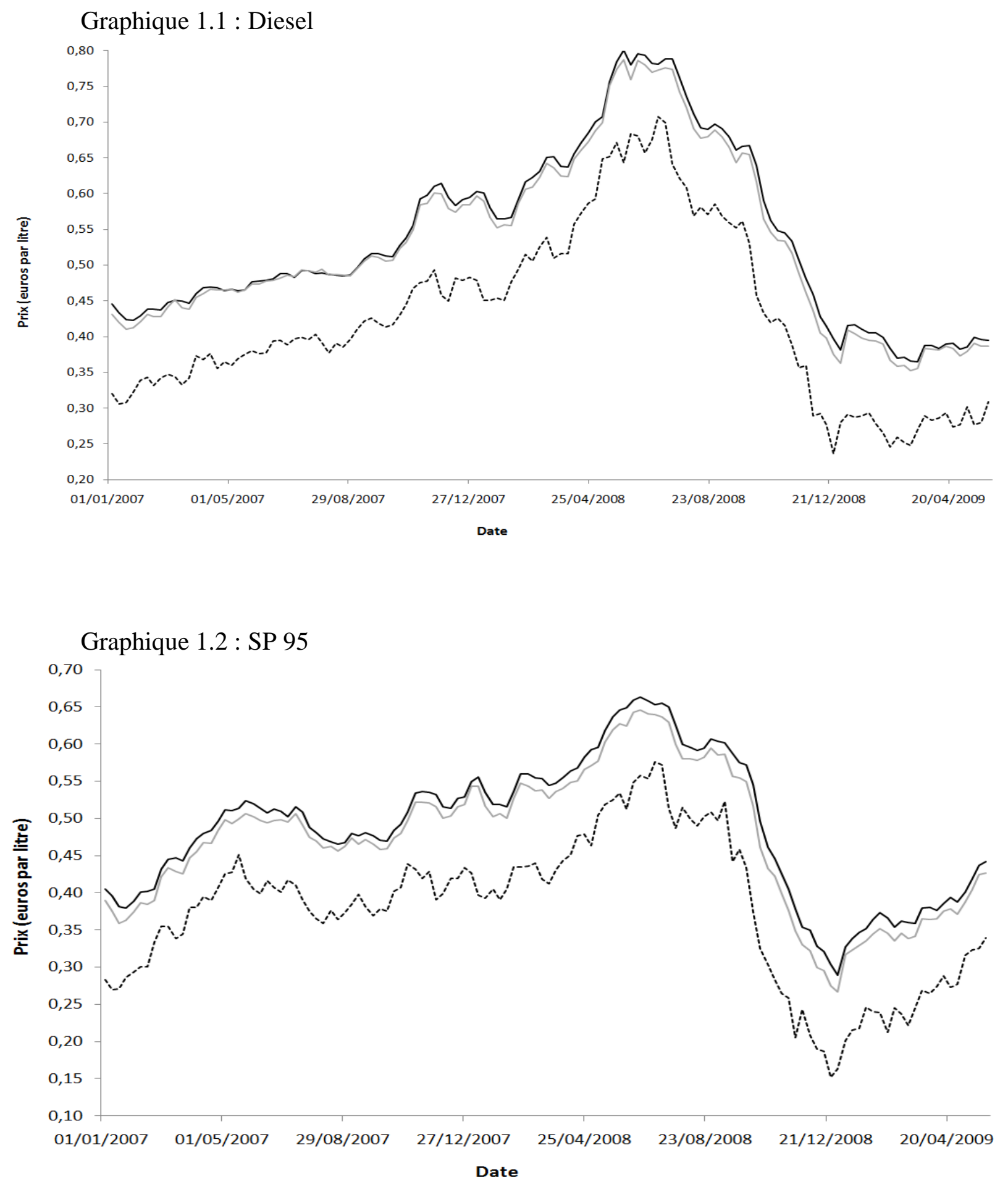

Lecture : La ligne en pointillé représente le prix de marché du produit raffiné à Rotterdam, la ligne noire les prix moyens hebdomadaires calculés à partir des relevés individuels de notre base de données et la ligne grise les prix hebdomadaires publiés par le Ministère de l'Economie. Le $1^{\text {er }}$ janvier 2007, le prix d'un litre de diesel est d'environ 0,32 euro à Rotterdam alors que le prix hors taxe moyen d'un litre de diesel en France est légèrement inférieur à 0,45 euro.

Source: Données individuelles quotidiennes issues du site Internet www.prix-carburants.gouv.fr (ensemble des stationsservice, janvier 2007-juin 2009), Ministère français de l'économie et U.S. Energy Information Administration, calculs des auteurs. 
Après traitement des valeurs aberrantes et des erreurs de mesure, notre échantillon contient 10169 stations-service pour le diesel et 10013 stations-service pour le SP 95. Le graphique 1 représente, à fréquence hebdomadaire, pour le diesel et le SP 95, le prix HT moyen observé dans notre base de données, le prix HT moyen publié par le Ministère de l'Economie et le prix du produit raffiné coté à Rotterdam (issus du site internet de l'U.S. Energy Information Administration (EIA)). Le prix moyen obtenu à partir de nos relevés individuels et le prix publié par le Ministère de l'Economie peuvent différer pour au moins deux raisons : notre statistique n'est calculée qu'à partir de 10000 stations-service sur les près de 13000 existantes, et elle n'est pas pondérée par les parts de marché des réseaux de distribution. Toutefois, la corrélation observée entre les deux séries est supérieure à 0,99 . L'écart systématique entre les deux séries s'explique par la part de marché supérieure des supermarchés dont les prix sont inférieurs à la moyenne. Le prix moyen du diesel et du SP 95 présentent des variations temporelles importantes : les prix doublent presque entre janvier 2007 et juin 2008. Puis, les prix moyens diminuent fortement pour atteindre en janvier 2009 des niveaux plus faibles qu'en 2007. Les prix se stabilisent ensuite autour de ces niveaux. Enfin, nous observons des évolutions proches entre les prix de marché des produits finis cotés à Rotterdam et les prix moyens des carburants dans les stations-service. Le ratio du prix moyen des carburants sur le prix Rotterdam ${ }^{\text {viii }}$ est en moyenne de $78 \%$ pour le diesel et $76 \%$ pour l'essence, ce ratio varie relativement peu sur la période.

\section{Fréquence des changements de prix des carburants}

Les prix sont considérés comme rigides s'ils réagissent avec retard aux chocs économiques auxquels les entreprises font face. Sous l'hypothèse que les chocs sont fréquents voire continus, une manière simple de caractériser la rigidité des prix est donc d'étudier la durée pendant laquelle un prix reste fixe ou la fréquence à laquelle les prix sont modifiés.

\section{Mesure de la rigidité des prix et enjeux théoriques}

La littérature théorique sur la rigidité des prix considère différents modèles simples permettant d'expliquer pourquoi un prix ne réagit pas immédiatement aux chocs (Gautier (2009) pour une synthèse) : (i) le modèle de coût de menu ou d'ajustement (menu-cost) suppose que les détaillants ont un coût physique à modifier leur prix (liés à l'affichage, à la présence ou à la disponibilité du personnel, à la publication d'un catalogue, etc.). En présence d'un choc, le détaillant aura le choix entre payer ce coût de menu et ajuster son prix aux nouvelles conditions du marché, ou ne pas payer ce coût et son prix s'éloignera du prix qui aurait été observé sans coût d'ajustement. La décision de changer de prix dépendra alors de l'environnement économique de l'entreprise ; (ii) un autre type de modèle (dit de dépendance au temps) suppose que la décision de changement de prix est indépendante de l'environnement économique. Dans ce modèle, des contrats de durée fixe (Taylor, 1980) ou aléatoire (Calvo, 1983) empêchent les détaillants de modifier leur prix en réponse aux chocs $^{\text {ix }}$; (iii) enfin, des modèles plus récents associent des imperfections d'information aux coûts de menu (Woodford, 2009 ; Alvarez et al., 2011). Les entreprises ont un coût à acquérir l'information sur leur environnement économique si bien qu'elles ne révisent pas leur ensemble d'information tous les jours. Quand elles révisent leur ensemble d'information, elles choisissent de changer leur prix ou non en fonction de la taille du choc et de leur coût de menu. Selon l'importance relative des coûts d'information et des coûts d'ajustement, ces modèles permettent de reproduire les prédictions des modèles de dépendance au temps et celles des modèles de coût d'ajustement. 
Comme le soulignent Blinder et al. (1998), ces théories reposent sur des variables difficilement observables en pratique ${ }^{\mathrm{x}}$. Pour tester la pertinence relative de ces théories, la littérature empirique sur la rigidité des prix relie des faits stylisés obtenus à partir de prix individuels aux prédictions des modèles. C'est l'approche empirique que nous adoptons ici.

\section{Durée des prix et fréquence de changement de prix}

Le tableau 1 présente les résultats sur la fréquence et la durée moyenne des prix des carburants en France $^{\mathrm{xi}}$. La durée moyenne entre deux changements de prix est de 6,6 jours pour le diesel et de 7 jours pour le SP 95. Chaque jour, 16,5\% des prix du SP 95 et 17,6\% des prix du diesel sont modifiés en moyenne. Les prix des carburants changent donc à une fréquence inférieure à celle des prix du pétrole brut. Pour les Etats-Unis, Davis et Hamilton (2004) et Douglas et Herrera (2010) estiment la durée moyenne des prix de gros des carburants à 3 jours alors que Davis (2007) obtient une fréquence des changements de prix de détail des carburants proche de $10 \%$.

Tableau 1 : Durée entre deux changements de prix et fréquence des changements de prix

\begin{tabular}{lccc}
\hline \hline & Ensemble & Supermarchés & Autres distributeurs \\
\hline Diesel & & & \\
Durée moyenne (en jours) & 6,64 & 6,01 & 7,14 \\
Fréquence (en \%) & & & \\
$\quad$ - Changements & 17,62 & 20,10 & 75,60 \\
$\quad$ - Hausses & 8,71 & 9,89 & 7,74 \\
$\quad$ - Baisses & 8,91 & 10,21 & \\
\hline SP 95 & & & 7,46 \\
Durée moyenne (en jours) & 7,02 & 6,47 & \\
Fréquence (en \%) & & & 14,86 \\
$\quad$ - Changements & 16,52 & 18,54 & 7,96 \\
$\quad$ - Hausses & 8,74 & 9,70 & 6,90 \\
$\quad$ - Baisses & 7,78 & 8,84 & \\
\hline \hline
\end{tabular}

Lecture : La durée est calculée comme le nombre de jours entre deux changements de prix ; nous calculons la moyenne de ces durées pour chaque station-service. Pour chaque station-service, une fréquence de changements est calculée comme le nombre de changements de prix sur le nombre total d'observations. Le tableau présente les moyennes des durées et fréquences calculées par station-service. Pour le diesel, la durée moyenne entre deux changements de prix est de 6,64 jours alors qu'en moyenne, $17,6 \%$ des prix changent chaque jour.

Source: Données individuelles quotidiennes issues du site internet www.prix-carburants.gouv.fr (ensemble des stations service disponibles, janvier 2007-juin 2009), calculs des auteurs.

La fonction de hasard fait partie des outils empiriques permettant d'évaluer la pertinence des différents modèles de rigidité de prix. Elle est définie comme la probabilité que la trajectoire de prix s'arrête au bout d'un certain nombre de périodes sachant que ce prix a survécu jusque là. Dans le modèle à coût de menu et avec une tendance d'inflation, le hasard sera croissant alors que dans le modèle de Calvo (avec probabilité exogène de changer les prix), le hasard sera constant quelle que soit la nature des chocs. S'il existe des contrats de durée fixe (Taylor, 1980), le hasard présentera des pics à certaines durées. Enfin, les modèles 
associant rigidité informationnelle et coûts de menu permettent d'obtenir des formes plus flexibles de fonctions de hasard (Woodford, 2009). Le graphique 2 représente les fonctions de hasard pour les prix du diesel et du SP 95. Les fonctions sont semblables dans les deux cas : elles présentent deux «paliers », un entre 1 et 7 jours puis un autre entre 8 et 15 jours, plus faible, alors qu'au-delà de 15 jours le hasard est légèrement croissant. Plusieurs pics significatifs apparaissent aux durées 7, 14 et 21 jours qui soulignent la régularité temporelle des changements de prix ${ }^{\text {xii }}$. Ces pics pourraient être cohérents avec la présence de contrats de prix fixes d'une ou plusieurs semaines. De tels contrats pourraient par exemple exister entre un fournisseur et la station-service afin de couvrir cette dernière contre les fluctuations du prix du pétrole. Nakamura et Steinsson (2011) ou Rotemberg (2005) mettent aussi en avant le rôle des relations entre détaillants et clients. S'il existe des habitudes de consommation et une information imparfaite des consommateurs sur les coûts, les détaillants peuvent avoir intérêt à s'engager sur un prix fixe, éviter de modifier trop souvent leur prix et ainsi minimiser les coûts d' «antagonisme» des clients. Nakamura et Steinsson (2011) montrent théoriquement que ce comportement aboutirait à des contrats implicites de prix rigides entre consommateurs et détaillants. Une autre interprétation relie ce résultat aux coûts informationnels de l'entreprise. L'acquisition de l'information sur les prix peut représenter un coût pour les stations-services ${ }^{\text {xiii }}$ associé à la chaîne de décision (siège de l'entreprise jusqu'à la stationservice) ou au temps de la décision sur les nouveaux prix qui peut impliquer des simulations par exemple (Zbaracki et al., 2004). Les entreprises ne révisent donc pas leur ensemble d'information tous les jours mais seulement certains jours particuliers de la semaine. 


\section{Graphique 2 : Fonctions de hasard pour les changements de prix}

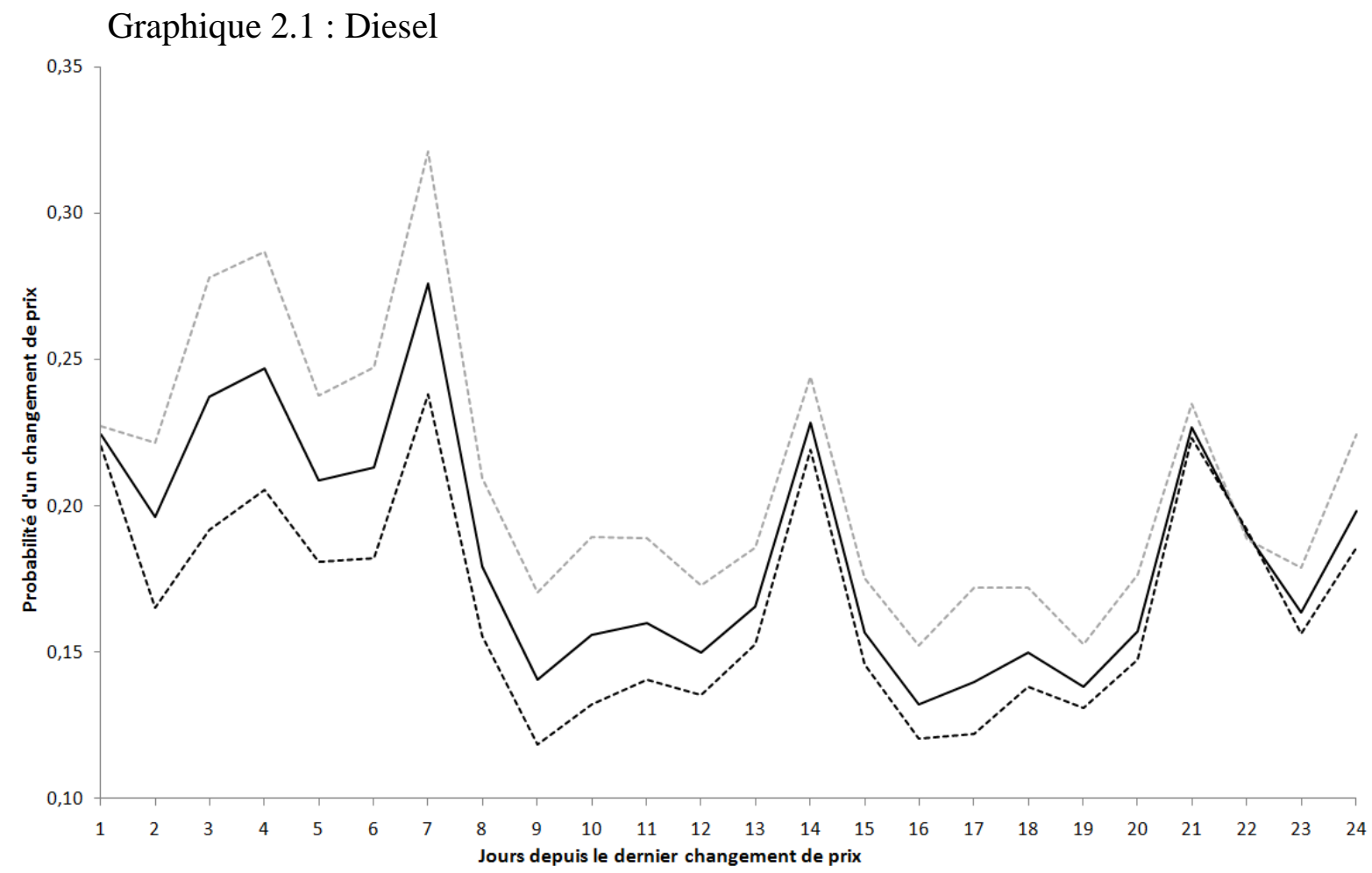

Graphique 2.2 : SP 95

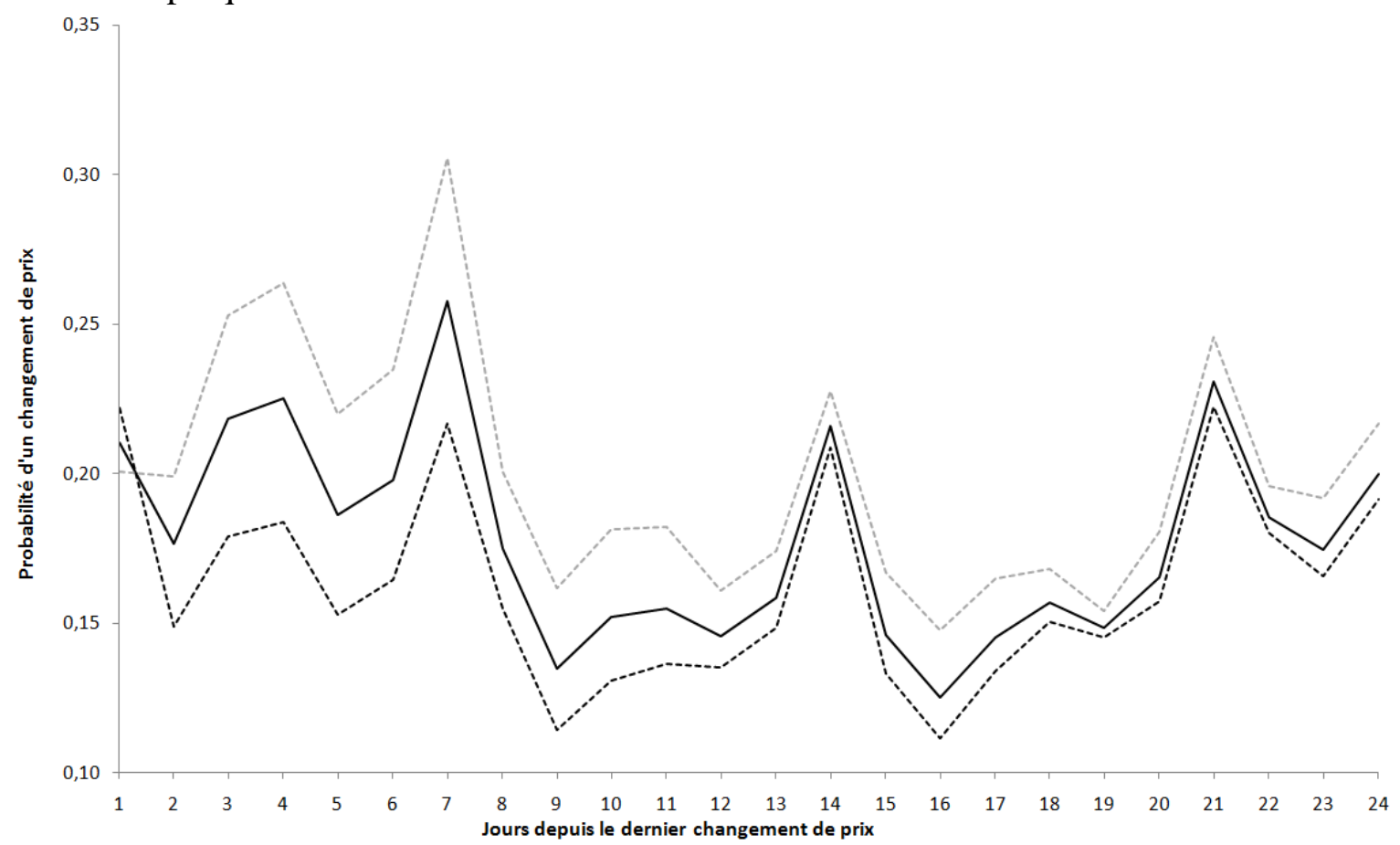

Lecture : Lignes pleines noires pour l'ensemble des stations-service, lignes grises en pointillés pour les supermarchés, lignes noires en pointillés pour les autres distributeurs. Le hasard est défini comme la probabilité que la trajectoire de prix s'arrête au bout d'un certain nombre de périodes sachant que ce prix a survécu jusque là. Les trajectoires de prix censurées à gauche sont exclues. Toutes les autres trajectoires de prix sont prises en compte. Par exemple, pour le diesel, parmi les prix ayant été modifiés il y a 6 jours et n'ayant pas encore été modifiés, un peu plus de $25 \%$ de ces prix sont changés au bout de 7 jours.

Source: Données individuelles quotidiennes issues du site internet www.prix-carburants.gouv.fr (ensemble des stations service disponibles, janvier 2007-juin 2009), calculs des auteurs. 


\section{Hétérogénéité de la fréquence des changements de prix}

Le diesel et le SP 95 sont deux produits relativement homogènes. En outre, un des chocs principaux sur le coût à distribuer le carburant vient du prix de la matière première et ce choc est commun à l'ensemble des stations-service. Pourtant, les durées moyennes par station-service sont hétérogènes. Le graphique 3 représente la distribution des durées individuelles entre deux changements de prix. La médiane est proche de 6 jours pour le diesel et le SP 95 mais un quart des durées sont inférieures à 5 jours et un quart supérieures à 8 jours.

\section{Graphique 3 : Distribution des durées moyennes des prix par station-service (en jours)}

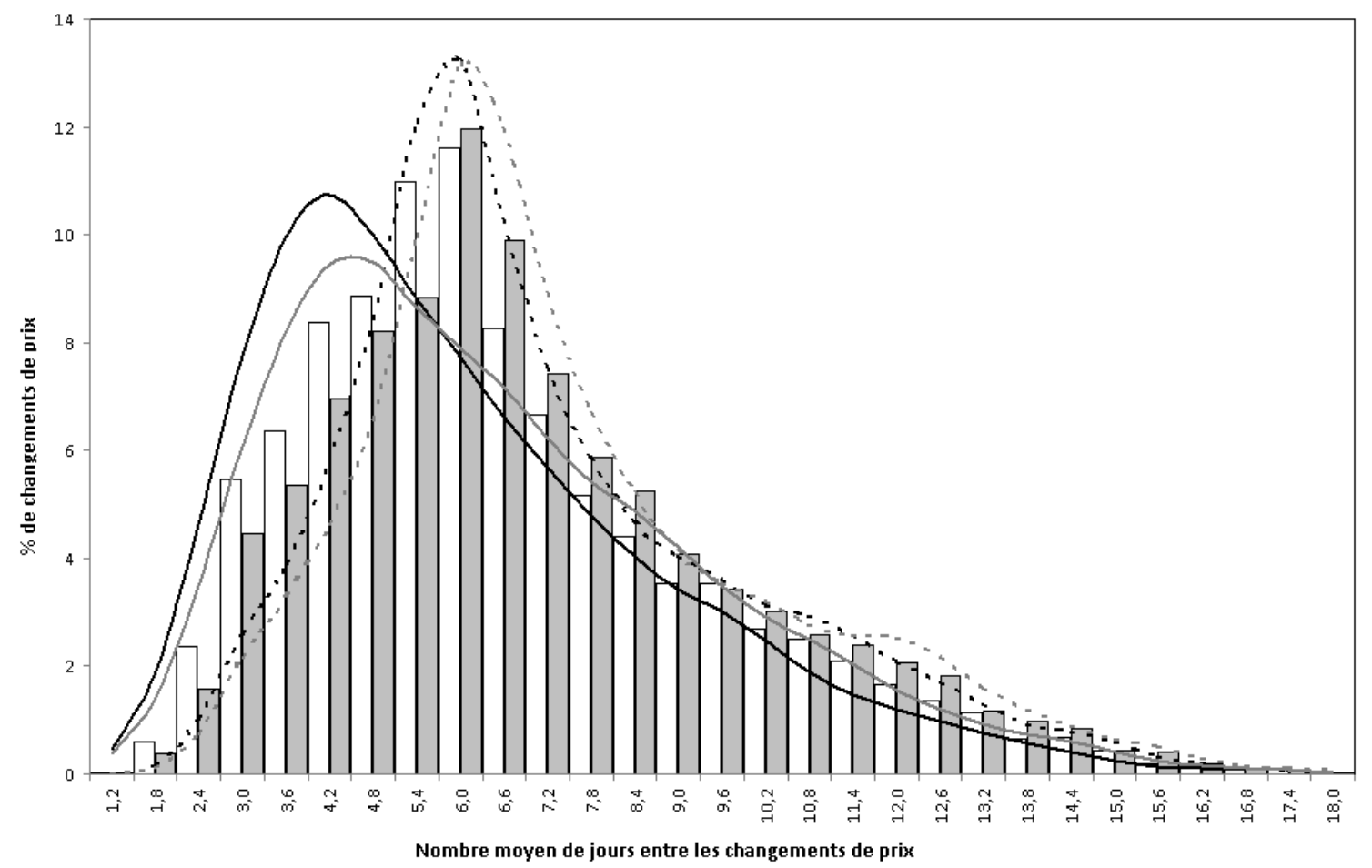

Lecture : Chaque observation est la durée moyenne entre deux changements de prix calculée pour chaque station-service. Les barres et lignes grises sont pour les prix du SP 95 et, les barres blanches et les lignes noires pour les prix du diesel. Les histogrammes sont pour l'ensemble des stations-service, les lignes pleines sont les estimateurs à noyau de la densité de la distribution des durées pour les supermarchés, les lignes en pointillés sont les estimateurs à noyau de la densité de la distribution des durées pour les autres distributeurs. Près de $12 \%$ des stations service ont une durée moyenne entre deux changements de prix de 6 jours (diesel ou SP95), cette proportion est plus proche de $8 \%$ pour les supermarchés et $14 \%$ pour les autres distributeurs.

Source: Données individuelles quotidiennes issues du site internet www.prix-carburants.gouv.fr (ensemble des stations service disponibles, janvier 2007-juin 2009), calculs des auteurs.

Afin de comprendre les déterminants de la fréquence des changements de prix par station-service, nous estimons un modèle empirique simple reliant la fréquence moyenne de changements de prix par station-service et des variables explicatives sur la concurrence et la structure de la demande locale. Rotemberg et Saloner (1987) montrent ainsi que la rigidité des prix diminue avec le niveau de la concurrence. Toutefois, peu de résultats empiriques permettent de valider cette prédiction. Zimmerman (2012) et Bellec et al. (2012) ont récemment souligné le rôle de la grande distribution sur la concurrence et le niveau des prix des carburants. Nous incluons une trentaine de variables indicatrices pour la marque de la station et évaluons un effet «supermarchés » ${ }^{\text {xiv }}$. Afin de mesurer le degré de concurrence, nous introduisons par ailleurs le nombre de stations-service dans un rayon de $10 \mathrm{kms}^{\mathrm{xv}}$ (en distinguant s'il s'agit de stations de supermarchés ou d'autres types de stations) et la distance 
à la station la plus proche selon que le plus proche concurrent est un supermarché ou une autre station. De même, la structure de la demande aura des conséquences sur la rigidité des prix dans la mesure où la réaction des consommateurs aux changements de prix pourrait être différente selon leurs caractéristiques démographiques. Enfin, nous considérons une variable indicatrice indiquant si la station-service a l'habitude d'utiliser des prix finissant en « 0 » ou « 9 ». Une littérature récente s'est en effet attachée à montrer que l'existence de prix dits psychologiques peut permettre d'expliquer la rigidité des prix. Knotek (2010) propose ainsi un modèle théorique montrant que l'adoption de prix psychologiques accroit le degré de rigidité des prix et qu'il est une alternative aux coûts de menu pour expliquer la rigidité des prix. Comme pour un coût de menu, les entreprises attendent que la taille des chocs soit suffisante pour changer leur prix d'un prix psychologique vers un autre. Enfin, nous ajoutons aussi des variables indicatrices pour le département dans lequel se situe la station-service. 
Tableau 2 : Déterminants de la fréquence de changement de prix par station-service

\begin{tabular}{|c|c|c|c|c|}
\hline & \multicolumn{2}{|c|}{ Diesel } & \multicolumn{2}{|c|}{ SP 95} \\
\hline & $f+$ & $f$ - & $f+$ & $f-$ \\
\hline $\begin{array}{l}\text { Distance à la plus proche station-service }(\mathrm{km}) \mathrm{x} \\
\text { le concurrent le plus proche est un supermarché }\end{array}$ & $\begin{array}{c}-0,093 * * * \\
(0,018)\end{array}$ & $\begin{array}{l}-0,118 * * * \\
(0,019)\end{array}$ & $\begin{array}{l}-0,078 * * * \\
(0,018)\end{array}$ & $\begin{array}{c}-0,097 * * * \\
(0,019)\end{array}$ \\
\hline $\begin{array}{l}\text { Distance à la plus proche station-service }(\mathrm{km}) \mathrm{x} \\
\text { le concurrent le plus proche n'est pas un supermarché }\end{array}$ & $\begin{array}{c}-0,048 * * \\
(0,019)\end{array}$ & $\begin{array}{l}-0,071 * * * \\
(0,020)\end{array}$ & $\begin{array}{c}-0,038^{* *} \\
(0,019)\end{array}$ & $\begin{array}{c}-0,055^{* * *} \\
(0,019)\end{array}$ \\
\hline $\begin{array}{l}\text { Nombre de stations-service d'un supermarché à moins } \\
\text { de } 10 \mathrm{kms}\end{array}$ & $\begin{array}{c}0,038 * * * \\
(0,008)\end{array}$ & $\begin{array}{c}0,034 * * * \\
(0,008)\end{array}$ & $\begin{array}{c}0,042 * * * \\
(0,008)\end{array}$ & $\begin{array}{c}0,036^{* * *} \\
(0,008)\end{array}$ \\
\hline $\begin{array}{l}\text { Nombre de stations-service d'un autre type à moins de } \\
10 \mathrm{kms}\end{array}$ & $\begin{array}{c}0,005 * * * \\
(0,002)\end{array}$ & $\begin{array}{c}0,005^{* *} \\
(0,002)\end{array}$ & $\begin{array}{c}0,006 * * * \\
(0,002)\end{array}$ & $\begin{array}{c}0,006 * * * \\
(0,002)\end{array}$ \\
\hline Autoroute & $\begin{array}{c}-0,457 * * * \\
(0,120)\end{array}$ & $\begin{array}{c}-0,442^{* * *} \\
(0,135)\end{array}$ & $\begin{array}{l}-0,166 \\
(0,118)\end{array}$ & $\begin{array}{c}-0,264 * * \\
(0,124)\end{array}$ \\
\hline Affichage stratégique des prix & $\begin{array}{c}-1,725 * * * \\
(0,075)\end{array}$ & $\begin{array}{l}-1,822 * * * \\
(0,081)\end{array}$ & $\begin{array}{l}-1,607 * * * \\
(0,073)\end{array}$ & $\begin{array}{c}-1,654 * * * \\
(0,076)\end{array}$ \\
\hline Aire rurale ou urbaine de moins de 20000 habitants & Ref & Ref & Ref & Ref \\
\hline $\begin{array}{l}\text { Agglomération de plus de } 20000 \text { à } 100000 \text { habitants } \\
\text { (hors Paris) }\end{array}$ & $\begin{array}{c}0,279 * * \\
(0,112)\end{array}$ & $\begin{array}{c}0,300^{* *} \\
(0,127)\end{array}$ & $\begin{array}{c}0,258 * * \\
(0,108)\end{array}$ & $\begin{array}{c}0,391 * * * \\
(0,121)\end{array}$ \\
\hline $\begin{array}{l}\text { Agglomération de plus de } 100000 \text { habitants (hors } \\
\text { Paris) }\end{array}$ & $\begin{array}{c}0,421 * * \\
(0,181)\end{array}$ & $\begin{array}{c}0,484 * * \\
(0,205)\end{array}$ & $\begin{array}{c}0,449 * * \\
(0,179)\end{array}$ & $\begin{array}{c}0,499 * * * \\
(0,193)\end{array}$ \\
\hline Paris et petite couronne & $\begin{array}{l}-0,785 \\
(0,501)\end{array}$ & $\begin{array}{c}-0,632 \\
(0,538)\end{array}$ & $\begin{array}{l}-0,557 \\
(0,523)\end{array}$ & $\begin{array}{l}-0,319 \\
(0,534)\end{array}$ \\
\hline Part des ménages possédant une voiture (\%) & $\begin{array}{c}0,024 * * * \\
(0,009)\end{array}$ & $\begin{array}{c}0,026 * * * \\
(0,009)\end{array}$ & $\begin{array}{c}0,023 * * * \\
(0,009)\end{array}$ & $\begin{array}{c}0,026^{* * *} \\
(0,009)\end{array}$ \\
\hline Taux de chômage (\%) & $\begin{array}{c}0,019 \\
(0,016)\end{array}$ & $\begin{array}{c}0,024 \\
(0,017)\end{array}$ & $\begin{array}{c}0,013 \\
(0,016)\end{array}$ & $\begin{array}{c}0,014 \\
(0,017)\end{array}$ \\
\hline Supermarché & $\begin{array}{c}0,987 * * * \\
(0,113)\end{array}$ & $\begin{array}{c}1,008 * * * \\
(0,124)\end{array}$ & $\begin{array}{c}1,064 * * * \\
(0,112)\end{array}$ & $\begin{array}{c}1,031 * * * \\
(0,121)\end{array}$ \\
\hline Constante & $\begin{array}{c}6,345^{* * * *} \\
(0,915)\end{array}$ & $\begin{array}{c}6,180 * * * \\
(0,973)\end{array}$ & $\begin{array}{c}6,286^{* * * *} \\
(0,912)\end{array}$ & $\begin{array}{c}4,998 * * * \\
(0,943)\end{array}$ \\
\hline R2 ajusté & 0,432 & 0,430 & 0,416 & 0,408 \\
\hline Nombre d'observations & 8077 & 8077 & 7857 & 7857 \\
\hline
\end{tabular}

Lecture : Les colonnes contiennent les résultats des estimations par moindres carrés ordinaires. Les variables endogènes sont la fréquence de baisse $(f-)$ et la fréquence de hausse $(f+)$ de prix (en $\%)$, par station-service. "Autoroute » est une variable indicatrice égale à 1 si la station-service est présente sur autoroute. «Affichage stratégique des prix » est une variable indicatrice égale à 1 si plus de $95 \%$ des prix constatés dans une station-service se terminent par 0 ou 9 . Le taux de chômage et la part des ménages possédant une voiture sont issus du recensement de la population 2008. Les régressions incluent en variables de contrôle des indicatrices pour la marque et le département. L'effet «Supermarché » est calculé à partir des effets « marque » (i.e. la soustraction de la moyenne des effets individuels « supermarchés » et « autres types de stations »), l'écarttype étant obtenu par la delta-méthode. Les estimations sont corrigées de l'hétéroscédasticité à l'aide de la matrice robuste de White. Niveau de significativité : *** $1 \%, * * 5 \%, * 10 \%$.

Source : Données individuelles quotidiennes issues du site internet www.prix-carburants.gouv.fr (stations service présentes plus de 500 jours dans la base de données, janvier 2007-juin 2009) et Insee, calculs des auteurs.

Les résultats des régressions sont présentés dans le tableau $2^{\mathrm{xvi}}$. La structure concurrentielle joue un rôle positif significatif sur la fréquence de changements de prix. Tout 
d'abord, les fréquences de hausses et de baisses sont significativement plus importantes dans les supermarchés et la durée moyenne entre deux changements de prix y est plus faible (Tableau 1). Par ailleurs, plus la station-service est proche d'une autre station, plus la probabilité de changer de prix à la hausse ou la baisse est grande. Cet effet est près de deux fois plus fort si la station la plus proche est un supermarché. De même, le nombre de stationsservice (en particulier le nombre de supermarchés) dans le voisinage d'une station-service joue un rôle positif sur la fréquence de changements. Les effets sont similaires pour le diesel et le SP 95. Ces résultats suggèrent que la présence d'un supermarché dont les prix sont souvent plus faibles accroît la pression concurrentielle sur les stations-service proches et diminue la rigidité des prix. Néanmoins, ces effets restent quantitativement faibles. Ainsi, avoir 5 stations-service d'un supermarché dans un rayon de $10 \mathrm{kms}$ augmente la fréquence de changement de prix d'environ 0,4 points de pourcentage (pp). Enfin, les stations sur autoroute changent moins souvent leur prix toutes choses égales par ailleurs. Sur des données américaines de prix de carburants, Hosken et al. (2008) ne trouvent pas d'effet significatif de la concurrence sur les prix. Sur l'ensemble des prix à la consommation aux Etats-Unis, Bils et Klenow (2004) obtiennent un effet positif mais non-significatif de la concurrence sur la fréquence de changements de prix.

Le nombre d'habitants (hors Paris) a un effet positif sur la fréquence de changements de prix (identique à la hausse et à la baisse). Plus la densité de la population est importante, plus les prix changent fréquemment. De même, la part des ménages disposant d'une voiture a un effet positif significatif. Les prix sont moins rigides et plus réactifs aux chocs dans les régions les plus densément peuplées parce que la demande serait plus importante ou l'information des consommateurs meilleure, les distances entre stations-service étant plus faibles. Hosken et al. (2008) trouvent un effet significatif similaire aux États-Unis.

Enfin, la présence de prix «psychologiques » a un effet négatif sur la durée entre deux changements de prix. Le tableau 3 présente les caractéristiques des durées de changements pour chaque dernier chiffre. Les prix des carburants sont habituellement affichés avec trois décimales dans les stations-service, et les prix sont collectés avec trois décimales. La distribution de la dernière décimale des prix TTC n'est cependant pas uniforme : $31 \%$ des prix se terminent par « $9 », 29 \%$ par « $0 », 9 \%$ par « $5 », 7 \%$ par « $4 »$ alors que pour les autres chiffres la proportion est inférieure à $5 \%$. Au contraire, la distribution de l'avant dernier chiffre apparaît uniforme. La durée moyenne pour les prix se terminant en « $0 »$ ou « 9 » est supérieure à celles des prix se terminant par un autre chiffre pour le diesel et le SP 95. Toutes choses égales par ailleurs, l'effet sur la fréquence de changement par stationservice est toujours très négatif et significatif (Tableau 2). Cet effet est identique pour les hausses et les baisses de prix. Levy et al. (2011) produisent des résultats similaires pour un ensemble de produits aux États-Unis. 
Tableau 3 : Durée entre deux changements de prix et taille moyenne des changements de prix en fonction du dernier chiffre du prix (incluant les taxes)

\begin{tabular}{ccccccc}
\hline \hline & $\begin{array}{c}\text { Diesel } \\
\text { \% des } \\
\text { trajectoires }\end{array}$ & $\begin{array}{c}\text { Durée } \\
(\text { en jours })\end{array}$ & $\begin{array}{c}|d p| \\
(\text { en } \%)\end{array}$ & $\begin{array}{c}\text { \% des 95 } \\
\text { trajectoires }\end{array}$ & $\begin{array}{c}\text { Durée } \\
\text { (en jours })\end{array}$ & $\begin{array}{c}|d p| \\
(\text { en \%) }\end{array}$ \\
\hline $\mathbf{0}$ & 29,0 & 6,7 & 2,63 & 29,5 & 7,0 & 3,13 \\
$\mathbf{1}$ & 2,9 & 4,2 & 2,22 & 3,0 & 4,6 & 2,79 \\
$\mathbf{2}$ & 4,2 & 4,1 & 2,05 & 4,2 & 4,4 & 2,58 \\
$\mathbf{3}$ & 3,6 & 4,3 & 2,17 & 3,6 & 4,7 & 2,73 \\
$\mathbf{4}$ & 7,2 & 4,2 & 1,97 & 7,3 & 4,4 & 2,44 \\
$\mathbf{5}$ & 8,8 & 4,5 & 2,23 & 8,8 & 4,9 & 2,78 \\
$\mathbf{6}$ & 3,7 & 4,4 & 2,19 & 3,8 & 4,7 & 2,74 \\
$\mathbf{7}$ & 4,1 & 4,4 & 2,16 & 3,9 & 4,7 & 2,67 \\
$\mathbf{8}$ & 4,7 & 4,7 & 2,28 & 4,6 & 5,0 & 2,84 \\
$\mathbf{9}$ & 31,8 & 4,9 & 2,40 & 31,4 & 5,3 & 2,94 \\
\hline \hline
\end{tabular}

Lecture : Nous considérons les prix incluant les taxes, la proportion des trajectoires de prix est calculée comme le ratio du nombre de trajectoires dont le prix se termine par un chiffre donné sur le total des trajectoires de prix et nous calculons la durée moyenne associée. $|d p|$ est la taille moyenne prise en valeur absolue sur les prix hors taxes. Le dernier chiffre est celui du prix TTC après le changement. Par exemple, les prix dont la dernière décimale est « 0 » représentent $29 \%$ des trajectoires, leur durée moyenne est de 6,7 jours pour le diesel et la taille moyenne des changements pour ces prix est de 2,63\%.

Source: Données individuelles quotidiennes issues du site internet www.prix-carburants.gouv.fr (ensemble des stations service disponibles, janvier 2007-juin 2009), calculs des auteurs.

\section{Distribution de la taille des changements de prix des carburants}

Si la rigidité des prix est principalement caractérisée par la fréquence de changement de prix, la littérature empirique s'est récemment concentrée sur les caractéristiques de la distribution des tailles de changements de prix (Cavallo et Rigobon, 2011). Les modèles théoriques de rigidité des prix proposent en effet des prédictions assez différentes sur la forme de cette distribution. Dans le modèle de Calvo, la distribution des changements devrait avoir la même forme que la distribution des variations de coût marginal. Le modèle de coût de mеnu prédit que la distribution sera bimodale : quand la variation de coût marginal est faible, il est trop coûteux de changer de prix, et les petits changements de prix seront rares. Enfin, les modèles de coût de menu avec information imparfaite permettent des formes plus flexibles de distribution de changements de prix. Alvarez et al. (2011) montrent ainsi que selon les calibrations du coût de menu et du coût d'information, la distribution peut être plus ou moins bimodale. Cette partie présente les caractéristiques de la distribution des changements de prix et les relie aux prédictions des modèles théoriques.

\section{Taille des changements de prix}

En moyenne, les hausses de prix sont aussi fréquentes que les baisses : pour le diesel, chaque jour, 8,7\% des stations-service augmentent leur prix alors que 8,9\% des stationsservice les baissent ; pour le SP 95, la fréquence des baisses est légèrement plus faible que la fréquence des hausses $(7,8 \%$ contre $8,7 \%)$. Les résultats sont similaires pour les supermarchés et les autres distributeurs (Tableau 1). Les changements de prix HT sont de plus grande ampleur pour le SP 95 (2,9\% en valeur absolue) que pour le diesel (2,3\% en valeur 
absolue) (Tableau 4). Pour les prix TTC, les tailles moyennes de changements sont plus faibles proches de $1,3 \%$ et les différences de taille des changements entre diesel et SP 95 sont proches de 0. Enfin, les différences entre l'ampleur moyenne des hausses et celle des baisses sont faibles pour les prix TTC et HT.

Tableau 4 : Taille des changements de prix (en \%)

\begin{tabular}{llllll}
\hline \hline & \multicolumn{2}{c}{ Hors taxes } & \multicolumn{2}{c}{ Toutes taxes comprises } \\
\hline Diesel & & Moyenne & Médiane & Moyenne & Médiane \\
Hausses & & & & \\
& Ensemble & & & & \\
& Supermarchés & 2,33 & 1,77 & 1,26 & 0,94 \\
& Autres distributeurs & 2,46 & 1,82 & 1,30 & 0,96 \\
Baisses & 2,16 & 1,71 & 1,21 & 0,92 \\
& Ensemble & & & & \\
& Supermarchés & $-2,41$ & $-1,87$ & $-1,32$ & $-0,98$ \\
& Autres distributeurs & $-2,50$ & $-1,91$ & $-1,34$ & $-1,00$ \\
\hline SP 95 & & $-1,85$ & $-1,30$ & $-0,96$ \\
Hausses & & & & \\
& Ensemble & & & & \\
& Supermarchés & 2,86 & 2,04 & 1,19 & 0,83 \\
& Autres distributeurs & 2,60 & 2,22 & 1,25 & 0,88 \\
Baisses & 2,60 & 1,92 & 1,12 & 0,81 \\
& Ensemble & $-2,93$ & $-2,16$ & $-1,26$ & $-0,86$ \\
& Supermarchés & $-3,08$ & $-2,22$ & $-1,30$ & $-0,88$ \\
& Autres distributeurs & $-2,75$ & $-2,10$ & $-1,22$ & $-0,85$ \\
\hline
\end{tabular}

Lecture : Les changements de prix sont calculés en pourcentage pour l'ensemble de trajectoires individuelles de prix (hors taxes et toutes taxes comprises). La moyenne et la médiane des changements de prix sont calculées sur la distribution totale des changements de prix (en excluant les «0»). La hausse moyenne des prix hors taxes (parmi les changements) est de $2,33 \%$ pour le diesel et 2,86 pour le SP95.

Source: Données individuelles quotidiennes issues du site internet www.prix-carburants.gouv.fr (ensemble des stations service disponibles, janvier 2007-juin 2009), calculs des auteurs.

Le graphique 4 présente la distribution des changements de prix pour le diesel et le SP 95 (TTC et HT). Une caractéristique remarquable de la distribution des changements de prix des carburants est sa «bimodalité ». Les changements de prix HT compris entre $-2 \%$ et $2 \%$ sont relativement rares par rapport à une distribution de type «normal » ${ }^{x i i}$ alors que le creux est limité aux changements entre $-1 \%$ et $1 \%$ pour les prix TTC. Cette distribution bimodale est cohérente avec les prédictions d'un modèle simple de coût de menu (Golosov et Lucas, 2007 ; Alvarez et al., 2011). 
Graphique 4.1 : Diesel

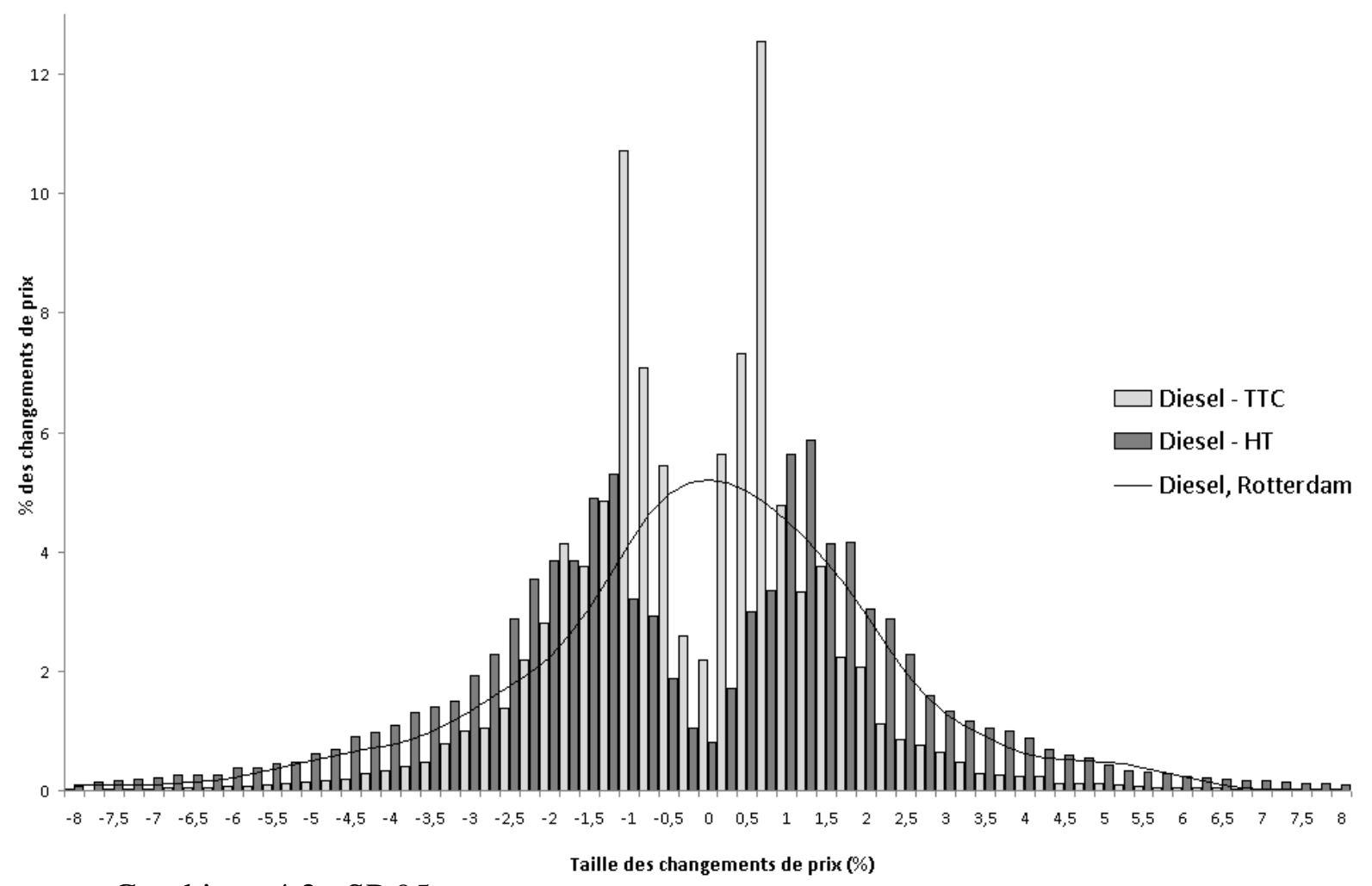

Graphique 4.2 : SP 95

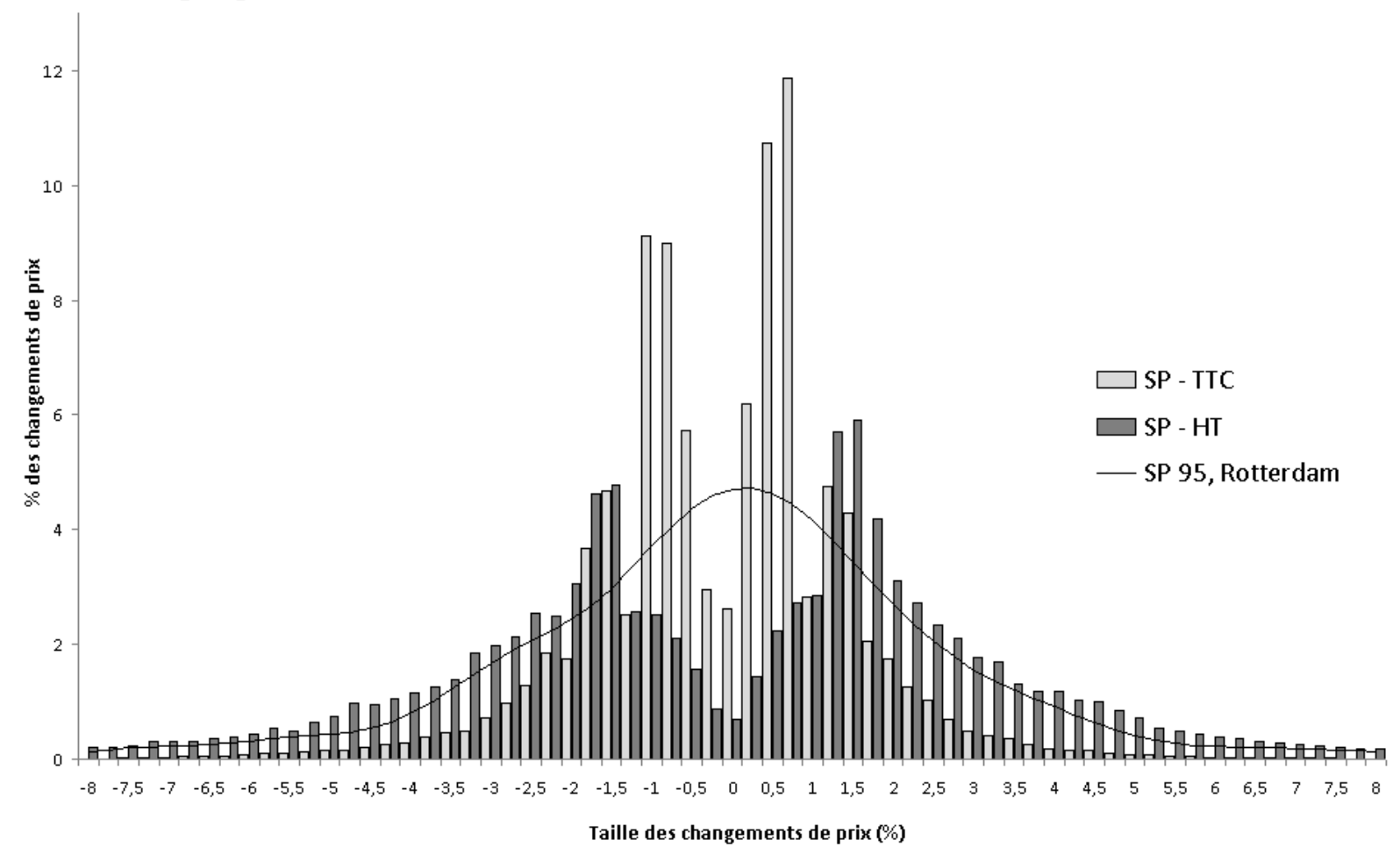

Lecture: Les observations sont les tailles des changements de prix individuels (calculées en \%) quand les prix sont effectivement changés (en excluant les changements égaux à 0). Les barres représentent la distribution des changements de prix des carburants (HT et TTC) et les lignes les estimateurs à noyau de la densité pour la distribution des changements de prix de marché à Rotterdam. 
Source: Données individuelles quotidiennes issues du site internet www.prix-carburants.gouv.fr (ensemble des stations service disponibles, janvier 2007-juin 2009), et U.S. Energy Information Administration, calculs des auteurs.

La faible proportion de petits changements de prix peut aussi être le résultat de chocs de grande taille sur le coût marginal. Sur le graphique, nous représentons la distribution des changements sur le prix du produit raffiné coté à Rotterdam qui est une approximation du coût de la matière première. Il apparaît que cette distribution est proche d'une loi normale avec une moyenne nulle. Le modèle de Calvo ne peut pas expliquer l'écart entre la distribution des variations de coûts et la distribution des changements de prix HT ou TTC.

Enfin, une dernière explication possible est liée au dernier chiffre du prix affiché. Dans la majorité des distributeurs les prix sont affichés sur trois chiffres après la virgule, ce qui implique qu'en principe, les petits changements de prix sont possibles. Par exemple, si le prix du diesel HT est de 0,450 euros, le gérant de la station-service peut modifier en théorie son prix de 0,001 euro et la taille de la hausse de prix HT serait alors de 0,22\%. Cependant, beaucoup de prix sont dits «psychologiques » ou «attractifs » et se terminent par un « $0 »$ ou un « $9 »$. Si le détaillant souhaite passer d'un prix se terminant en « $0 »$ à un autre prix se terminant en « $0 »$ alors le changement de prix en termes absolus est de 0,01 euro soit 2,2\%. Le graphique 5 représente la distribution des changements de prix pour les stations-service dont plus de $95 \%$ des prix se terminent par « $0 »$ ou « $9 »$ comparée à la distribution des changements des autres stations. La bimodalité est renforcée pour les stations-service avec des prix «attractifs » où aucun changement n'est inférieur à $1 \%$ en valeur absolue mais elle reste présente pour les autres stations-service. Les pics autour de 1 à $2 \%$ sont plus élevés pour les stations-service adoptant systématiquement des prix psychologiques. Cette bimodalité est aussi à relier avec les durées plus longues : le calendrier de changements de prix est modifié par la présence de prix psychologiques, les entreprises attendent plus longtemps avant de changer leur prix et opèrent donc des changements de plus grande ampleur (Tableau 3). 


\section{Graphique 5 : Stratégie d'affichage des prix et distribution des tailles de changements de prix (HT - en \%)}

Graphique 5.1 : Diesel

$5.1 .1 \ll$ Prix attractifs »

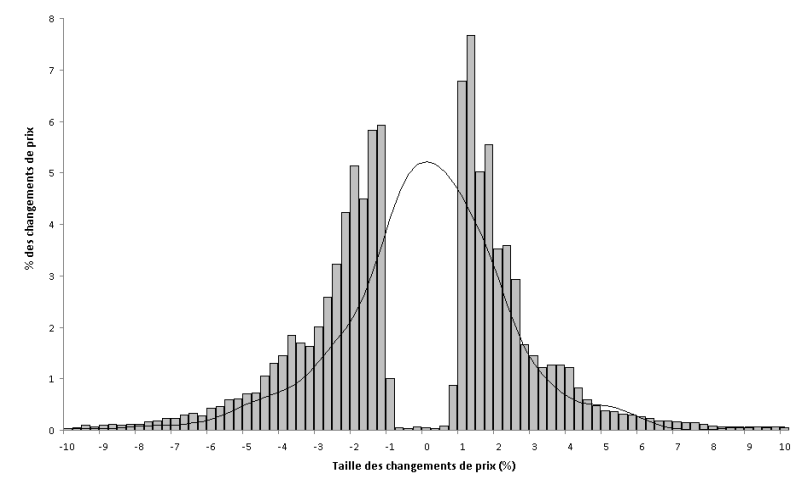

Graphique 5.2 : SP 95

5.2.1 « Prix attractifs »

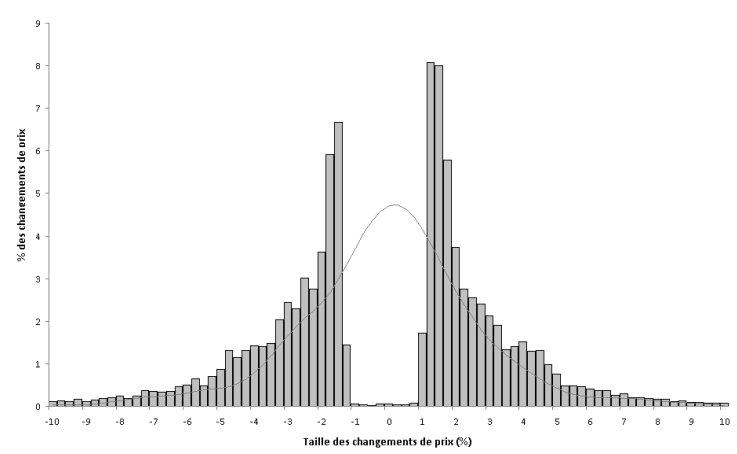

5.1 .2 «utres prix »

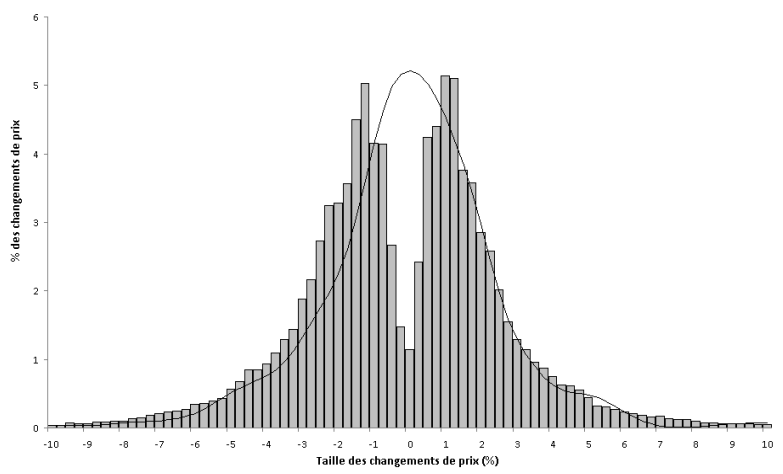

5.2.2 « Autres prix»

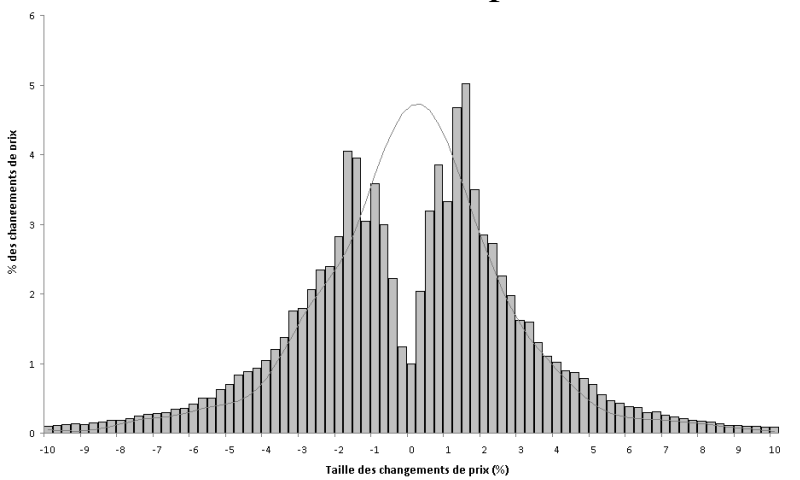

Lecture: Les observations sont les tailles des changements de prix individuels (calculées en \%) quand les prix sont effectivement changés (en excluant les changements égaux à 0). Les barres grises représentent la distribution des changements de prix des carburants et les lignes grises les estimateurs à noyau de la densité pour la distribution des changements de prix de marché à Rotterdam. Les changements de prix des carburants sont calculés à partir des prix hors taxes. L'affichage stratégique des prix correspond aux stations-service dont la proportion de prix se terminant par 0 ou 9 est supérieure à $95 \%$.

Source: Données individuelles quotidiennes issues du site internet www.prix-carburants.gouv.fr (ensemble des stations service disponibles, janvier 2007-juin 2009), et U.S. Energy Information Administration, calculs des auteurs.

\section{Hétérogénéité de la taille des changements de prix}

L'hétérogénéité entre stations pour la taille moyenne des changements de prix est moins marquée que pour la fréquence de changements. La taille moyenne des hausses de prix du diesel est de 2,5\% pour les supermarchés contre $2,2 \%$ pour les autres stations-service. Les écarts ne sont jamais supérieurs à $0,3 \mathrm{pp}$. L'écart entre supermarchés et autres stations est encore plus faible sur les prix TTC (proche de 0,1 pp) (Tableau 4). Les supermarchés changent donc leur prix plus souvent et par des changements un peu plus grands (en valeur absolue) que les autres stations-service. Des différences de structure de coût des stationsservice sont une explication possible à cette observation. On peut considérer schématiquement que les coûts de production des stations-service comprennent le coût de la matière première et d'autres coûts liés à la distribution (coût du travail, entretien de la station, etc.). Il est cependant possible que pour les supermarchés, les coûts autres que la matière première 
représentent une part plus faible des coûts totaux. La part du coût de la matière première serait alors plus grande pour les supermarchés que pour les autres stations ${ }^{\text {xviii }}$. Comme le prix de la matière première varie plus fréquemment que les autres coûts, la fréquence des changements de prix dans les supermarchés sera plus élevée, et les changements de prix répercuteront davantage les variations de prix de la matière première. De même, si le taux de marge des stations de supermarché est plus faible, les hausses et les baisses de prix seront plus fréquentes et plus importantes en moyenne.

Comme précédemment, nous utilisons une régression linéaire pour relier les tailles moyennes de hausses et de baisses de prix HT par station-service à des indicateurs de structure concurrentielle et de demande locale (Tableau 5). La fréquence et la taille des changements de prix sont négativement corrélées, ce qui peut s'expliquer par un effet de sélection caractéristique des modèles à coût d'ajustement. Pour les entreprises qui attendent moins (resp. plus) longtemps avant de changer de prix, la taille de changement sera plus faible (resp. importante). Le phénomène est accentué pour les stations-service avec des prix attractifs en « $0 »$ ou « 9 ». Cette observation est cohérente avec un modèle où fixer un prix psychologique s'apparente à un coût d'ajustement plus important. Au contraire, pour les stations des grandes agglomérations (hors Paris), les prix changent plus souvent mais les changements sont en moyenne plus petits. Enfin, les variables de concurrence n'ont pas d'effet significatif sur la taille des changements. Seule l'indicatrice «autoroute » a un effet significatif négatif : les prix des carburants sur autoroute changent moins souvent et la taille des changements est plus faible, ce qui pourrait s'expliquer par un poids plus faible du prix de la matière première dans la structure de coût.

Afin de tester cette hypothèse et de mesurer les déterminants des différences de niveau moyen des prix entre stations, nous calculons la différence moyenne entre le prix de vente d'une station-service (HT, en logarithme) et le prix du produit raffiné à Rotterdam (en logarithme) qui approxime le coût marginal ${ }^{\mathrm{xix}}$ (Tableau 5). Cette différence est utilisée par Davis et Hamilton (2004) ou Hosken et al. (2008) pour mesurer le taux de marge brut moyen de transport-distribution de chaque station-service. Elle inclut cependant les coûts logistiques (stockage, transport), d'exploitation (frais de personnel) et réglementaires (mise aux normes) que nous n'observons pas. Cette marge brute est significativement plus faible (-7 pp) pour les supermarchés et plus forte $(+9$ à $10 \mathrm{pp})$ pour les stations présentes sur autoroute. Cette hétérogénéité provient en partie d'importantes différences de coûts d'exploitation qui sont plus faibles pour les supermarchés que pour les stations d'autoroute. La structure concurrentielle joue aussi un rôle significatif sur les marges. Le nombre de supermarchés dans le voisinage d'une station réduit significativement la marge brute alors que la distance à la plus proche station-service voisine (que ce soit un supermarché ou une autre station) l'augmente. Enfin, la marge brute est plus faible pour les stations des grandes aires urbaines (hors Paris) mais plus forte à Paris où la concurrence est plus faible mais où les coûts d'exploitation sont aussi plus importants (loyers par exemple). 


\section{Tableau 5 : Déterminants des tailles de changement de prix et du taux de marge brut de transport-distribution par station-service}

\begin{tabular}{|c|c|c|c|c|c|c|}
\hline & \multicolumn{3}{|c|}{ Diesel } & \multicolumn{3}{|c|}{ SP 95} \\
\hline & $d p+$ & $|d p-|$ & $\begin{array}{l}\text { Taux de } \\
\text { marge }\end{array}$ & $d p+$ & $|d p-|$ & $\begin{array}{l}\text { Taux de } \\
\text { marge }\end{array}$ \\
\hline $\begin{array}{l}\text { Distance à la plus proche station-service }(\mathrm{km}) \mathrm{x} \\
\text { le concurrent le plus proche est un supermarché }\end{array}$ & $\begin{array}{c}0,002 \\
(0,003)\end{array}$ & $\begin{array}{l}0,007 * \\
(0,004)\end{array}$ & $\begin{array}{c}0,075 * * * \\
(0,016)\end{array}$ & $\begin{array}{c}0,001 \\
(0,004)\end{array}$ & $\begin{array}{c}0,008 \\
(0,005)\end{array}$ & $\begin{array}{c}0,122 * * * \\
(0,020)\end{array}$ \\
\hline $\begin{array}{l}\text { Distance à la plus proche station-service }(\mathrm{km}) \mathrm{x} \\
\text { le concurrent le plus proche n'est pas un } \\
\text { supermarché }\end{array}$ & $\begin{array}{l}-0,000 \\
(0,003)\end{array}$ & $\begin{array}{l}0,006^{*} \\
(0,004)\end{array}$ & $\begin{array}{c}0,114 * * * \\
(0,020)\end{array}$ & $\begin{array}{l}-0,003 \\
(0,004)\end{array}$ & $\begin{array}{c}0,006 \\
(0,005)\end{array}$ & $\begin{array}{c}0,122 * * * \\
(0,024)\end{array}$ \\
\hline $\begin{array}{l}\text { Nombre de stations-service d'un supermarché à } \\
\text { moins de } 10 \mathrm{kms}\end{array}$ & $\begin{array}{c}0,003 * * \\
(0,001)\end{array}$ & $\begin{array}{c}0,003^{* *} \\
(0,001)\end{array}$ & $\begin{array}{l}-0,072 * * * \\
(0,009)\end{array}$ & $\begin{array}{l}0,003^{*} \\
(0,002)\end{array}$ & $\begin{array}{l}0,003 * \\
(0,002)\end{array}$ & $\begin{array}{c}-0,072 * * * \\
(0,012)\end{array}$ \\
\hline $\begin{array}{l}\text { Nombre de stations-service d'un autre type à moins } \\
\text { de } 10 \mathrm{kms}\end{array}$ & $\begin{array}{l}-0,000 \\
(0,000)\end{array}$ & $\begin{array}{l}-0,000 \\
(0,000)\end{array}$ & $\begin{array}{c}0,008 * * * \\
(0,003)\end{array}$ & $\begin{array}{l}-0,000 \\
(0,000)\end{array}$ & $\begin{array}{l}-0,000 \\
(0,000)\end{array}$ & $\begin{array}{l}-0,003 \\
(0,004)\end{array}$ \\
\hline Autoroute & $\begin{array}{c}-0,643 * * * \\
(0,020)\end{array}$ & $\begin{array}{c}-0,655^{* * * *} \\
(0,021)\end{array}$ & $\begin{array}{l}9,805 * * * \\
(0,277)\end{array}$ & $\begin{array}{c}-0,729 * * * \\
(0,026)\end{array}$ & $\begin{array}{c}-0,760 * * * \\
(0,027)\end{array}$ & $\begin{array}{c}8,719 * * * \\
(0,272)\end{array}$ \\
\hline Affichage stratégique des prix & $\begin{array}{c}0,146^{* * *} \\
(0,015)\end{array}$ & $\begin{array}{c}0,196 * * * \\
(0,017)\end{array}$ & $\begin{array}{c}0,862 * * * \\
(0,096)\end{array}$ & $\begin{array}{c}0,150 * * * \\
(0,017)\end{array}$ & $\begin{array}{c}0,224 * * * \\
(0,021)\end{array}$ & $\begin{array}{c}1,534 * * * \\
(0,126)\end{array}$ \\
\hline Aire rurale ou urbaine de moins de 20000 habitants & Ref & Ref & Ref & Ref & Ref & Ref \\
\hline $\begin{array}{l}\text { Agglomération de plus de } 20 \quad 000 \text { à } 100 \quad 000 \\
\text { habitants (hors Paris) }\end{array}$ & $\begin{array}{c}-0,051 * * * \\
(0,016)\end{array}$ & $\begin{array}{l}-0,048 * * * \\
(0,018)\end{array}$ & $\begin{array}{l}-0,443 * * * \\
(0,112)\end{array}$ & $\begin{array}{l}-0,030 \\
(0,021)\end{array}$ & $\begin{array}{c}-0,052 * * \\
(0,023)\end{array}$ & $\begin{array}{c}-0,829 * * * \\
(0,143)\end{array}$ \\
\hline $\begin{array}{l}\text { Agglomération de plus de } 100000 \text { habitants (hors } \\
\text { Paris) }\end{array}$ & $\begin{array}{c}-0,083 * * * \\
(0,027)\end{array}$ & $\begin{array}{l}-0,054^{*} \\
(0,032)\end{array}$ & $\begin{array}{l}-0,613 * * * \\
(0,192)\end{array}$ & $\begin{array}{c}-0,080 * * \\
(0,033)\end{array}$ & $\begin{array}{l}-0,110^{* * *} \\
(0,039)\end{array}$ & $\begin{array}{c}-1,188 * * * \\
(0,263)\end{array}$ \\
\hline Paris et petite couronne & $\begin{array}{r}-0,150^{*} \\
(0,078)\end{array}$ & $\begin{array}{l}-0,030 \\
(0,085)\end{array}$ & $\begin{array}{r}1,015^{*} \\
(0,573)\end{array}$ & $\begin{array}{l}-0,159^{*} \\
(0,095)\end{array}$ & $\begin{array}{l}-0,068 \\
(0,095)\end{array}$ & $\begin{array}{c}3,092 * * * \\
(0,732)\end{array}$ \\
\hline Part des ménages possédant une voiture (\%) & $\begin{array}{c}-0,002 \\
(0,001)\end{array}$ & $\begin{array}{l}-0,002 \\
(0,002)\end{array}$ & $\begin{array}{c}-0,022 * * \\
(0,009)\end{array}$ & $\begin{array}{l}-0,003 * \\
(0,002)\end{array}$ & $\begin{array}{c}-0,005 * * \\
(0,002)\end{array}$ & $\begin{array}{l}-0,013 \\
(0,012)\end{array}$ \\
\hline Taux de chômage (\%) & $\begin{array}{l}-0,000 \\
(0,003)\end{array}$ & $\begin{array}{c}-0,003 \\
(0,003)\end{array}$ & $\begin{array}{l}-0,027 \\
(0,016)\end{array}$ & $\begin{array}{c}-0,002 \\
(0,003)\end{array}$ & $\begin{array}{l}-0,007^{*} \\
(0,004)\end{array}$ & $\begin{array}{l}-0,022 \\
(0,021)\end{array}$ \\
\hline Supermarché & $\begin{array}{c}0,456 * * * \\
(0,024)\end{array}$ & $\begin{array}{c}0,542 * * * \\
(0,026)\end{array}$ & $\begin{array}{c}-7,545^{* * *} \\
(0,152)\end{array}$ & $\begin{array}{c}0,672 * * * \\
(0,031)\end{array}$ & $\begin{array}{c}0,702 * * * \\
(0,036)\end{array}$ & $\begin{array}{c}-7,055^{* * *} * \\
(0,226)\end{array}$ \\
\hline Fréquence de hausses ou baisses & $\begin{array}{c}-0,121 * * * \\
(0,003)\end{array}$ & $\begin{array}{c}-0,136 * * * \\
(0,003)\end{array}$ & & $\begin{array}{c}-0,144 * * * \\
(0,003)\end{array}$ & $\begin{array}{c}-0,171 * * * \\
(0,004)\end{array}$ & \\
\hline Constante & $\begin{array}{c}3,257 * * * \\
(0,149)\end{array}$ & $\begin{array}{c}3,489 * * * \\
(0,166)\end{array}$ & $\begin{array}{c}35,124 * * * \\
(0,942)\end{array}$ & $\begin{array}{c}4,052 * * * \\
(0,182)\end{array}$ & $\begin{array}{c}4,392 * * * \\
(0,211)\end{array}$ & $\begin{array}{c}34,505 * * * \\
(1,228)\end{array}$ \\
\hline R2 ajusté & 0,616 & 0,651 & 0,853 & 0,584 & 0,625 & 0,751 \\
\hline Nombre d'observations & 8077 & 8077 & 8077 & 7857 & 7857 & 7857 \\
\hline
\end{tabular}

Lecture : Les colonnes contiennent les résultats des estimations par moindres carrés ordinaires. Les variables endogènes sont la moyenne des hausses $(d p+)$ et des baisses $(d p-)$ des prix HT (en \%) calculée par station service et le taux de marge moyen défini comme la différence moyenne du log du prix HT et du log du prix Rotterdam, par station-service. «Autoroute» est une variable indicatrice égale à 1 si la station-service est présente sur autoroute. "Affichage stratégique des prix » est une variable indicatrice égale à 1 si plus de $95 \%$ des prix constatés dans une station-service se terminent par 0 ou 9 . Le taux de chômage et la part des ménages possédant une voiture sont issus du recensement de la population 2008. Les régressions incluent en variables de contrôle des indicatrices pour la marque et le département. L'effet «Supermarché » est calculé à partir des effets «marque» (i.e. la soustraction de la moyenne des effets individuels «supermarchés» et «autres types de stations »), l'écart-type étant obtenu par la delta-méthode. Les estimations sont corrigées de l'hétéroscédasticité à l'aide de la matrice robuste de White. Niveau de significativité : *** $1 \%, * * 5 \%, * 10 \%$.

Source : Source : Données individuelles quotidiennes issues du site internet www.prix-carburants.gouv.fr (stations service présentes plus de 500 jours dans la base de données, janvier 2007-juin 2009) et Insee, calculs des auteurs. 
Les durées de prix les plus longues sont-elles associées aux changements les plus grands?

Le graphique 6 présente la relation entre la durée moyenne entre deux changements de prix et l'ampleur moyenne des changements en valeur absolue (ensemble, supermarchés et autres distributeurs). Pour le diesel comme pour le SP 95, nous obtenons une corrélation positive assez forte entre les deux variables, en particulier jusqu'aux durées inférieures à 10 jours. Pour les durées plus longues la relation semble s'affaiblir ${ }^{\mathrm{xx}}$. En outre, la corrélation est beaucoup plus forte pour les supermarchés que pour les autres stations, ce qui renforce la conclusion que les supermarchés répercutent davantage la volatilité des variations de coût marginal parce que la part de la matière première dans leurs coûts est plus importante. Par comparaison, Baudry et al. (2005) obtiennent une corrélation faible entre taille et fréquence pour l'ensemble des prix à la consommation. De même sur données américaines, Klenow et Kryvtsov (2008) soulignent l'absence de corrélation entre durée depuis le dernier changement de prix et taille des changements.

Dans un modèle de coût d'ajustement simple et avec une inflation tendancielle, l'effet de sélection produira une corrélation positive entre la taille du changement de prix et la durée depuis le dernier changement de prix : les entreprises qui changent leur prix sont celles qui se sont le plus éloignées de leur prix optimal sans rigidité et aussi celles qui ont attendu le plus longtemps avant de changer leur prix (Golosov et Lucas, 2007). Ce ne sera pas le cas dans le modèle de Calvo et nos observations tendent donc à invalider ce modèle. Par ailleurs, dans un modèle associant coût de menu et coût d'information, la corrélation sera d'autant plus importante que le coût prépondérant est le coût d'ajustement (Woodford, 2009). 
Graphique 6 : Taille moyenne des changements de prix (HT - en \% - valeur absolue) en fonction de la durée depuis le dernier changement de prix (en jours)

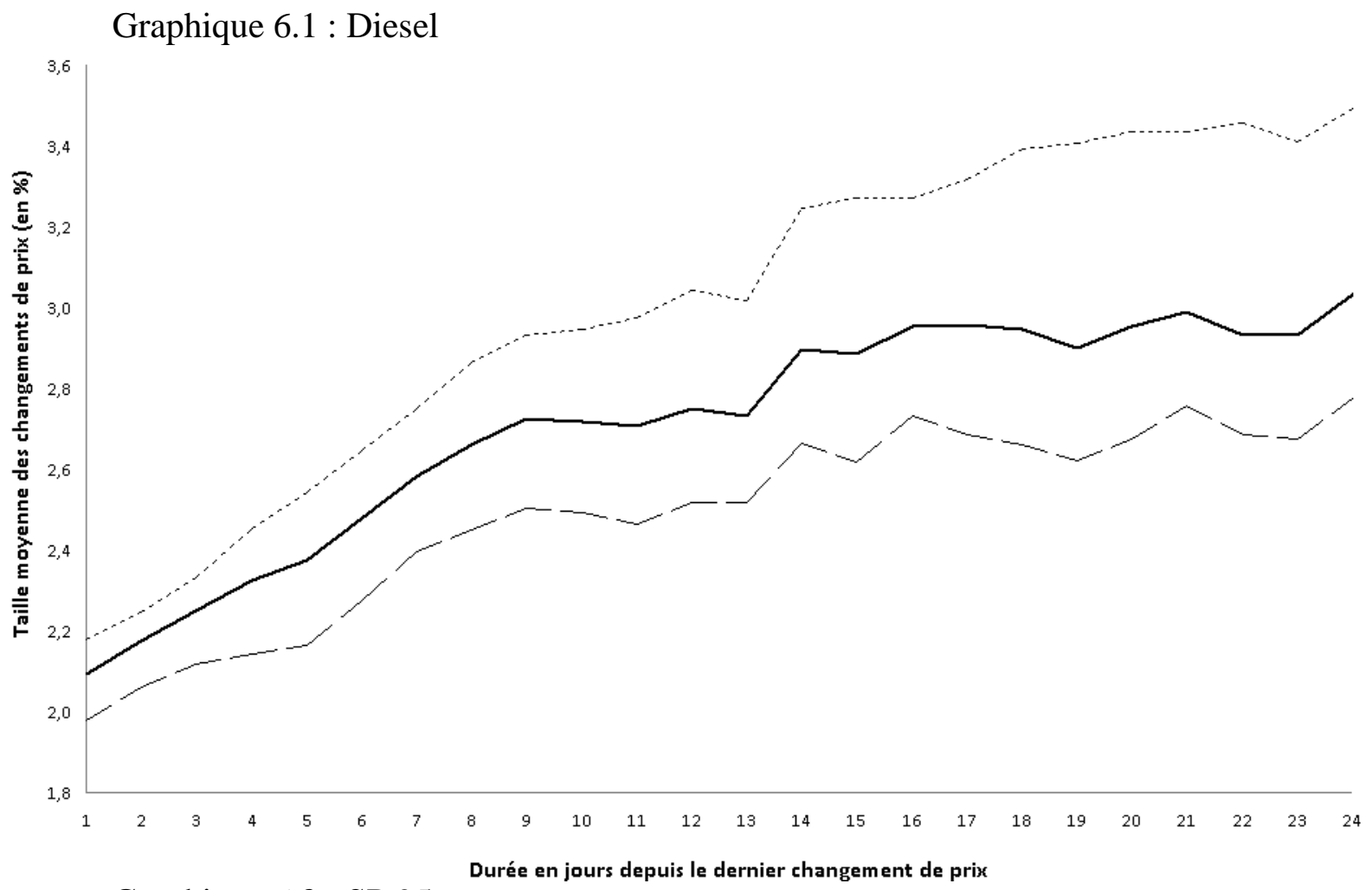

Graphique 6.2 : SP 95

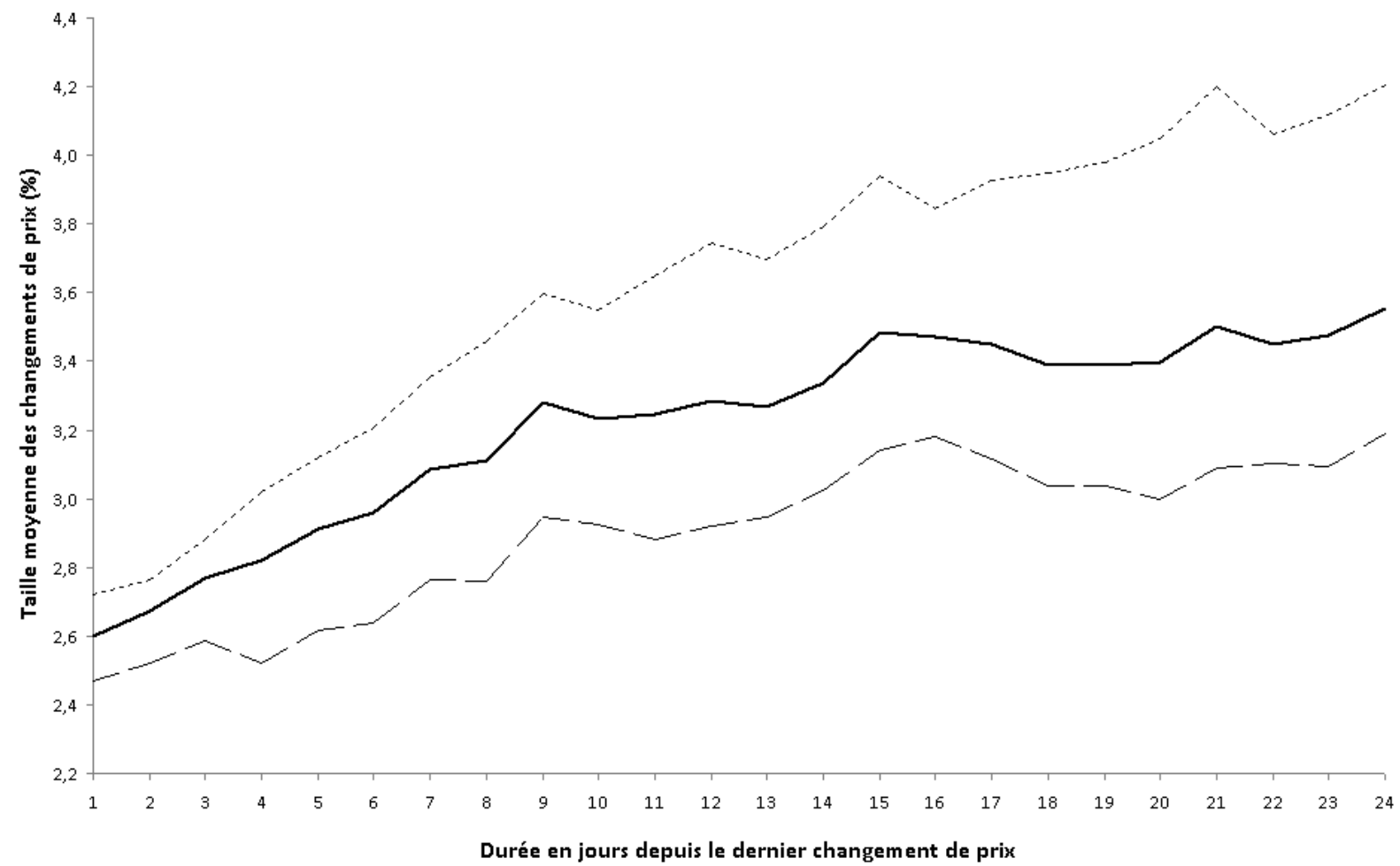

Lecture : Pour l'ensemble des trajectoires de prix, nous calculons la taille moyenne (en valeur absolue) des changements de prix associée à chaque durée de prix. La taille des changements de prix des carburants est calculée à partir des prix hors taxes. Les lignes noires pleines sont pour l'ensemble des stations-service, les lignes en petits pointillés pour les supermarchés, les lignes en grands pointillés pour les autres distributeurs. Pour le diesel, la taille moyenne des changements de prix intervenant une journée après le dernier changement est de $2,1 \%$. 
Source: Données individuelles quotidiennes issues du site internet www.prix-carburants.gouv.fr (ensemble des stations service présentes dans la base de données, janvier 2007-juin 2009), calculs des auteurs.

\section{Rigidité des prix et variations de l'inflation des prix des carburants}

Dans cette partie, nous étudions les variations temporelles de la fréquence et de la taille des changements de prix et rapprochons nos résultats des prédictions des modèles théoriques. Dans le modèle de Calvo, la fréquence de changements de prix est supposée constante dans le temps et prédéterminée, l'inflation varie parce que la taille des changements varie dans le temps. Le modèle de coût d'ajustement suppose lui que la décision de changement de prix est endogène, et l'inflation varie parce que la fréquence de changements de prix varie fortement en fonction de l'environnement économique.

\section{Dynamique de l'inflation des prix des carburants}

Klenow et Kryvtsov (2008) ont récemment proposé un exercice de décomposition de l'inflation entre marge intensive - la taille moyenne des changements de prix (parmi les détaillants ayant modifié leurs prix) - et marge extensive - la fréquence des changements de prix. L'inflation est en effet le résultat de l'agrégation de comportements individuels d'ajustement des prix : elle peut augmenter parce plus de détaillants augmentent leur prix ou parce que les détaillants quand ils changent leur prix, augmentent davantage leur prix. La fréquence et la taille moyenne des changements de prix au cours du temps vont donc influer la dynamique agrégée de l'inflation. Nous utilisons ensuite ces résultats pour les confronter aux prédictions des modèles théoriques de rigidité des prix.

Une approximation de l'inflation à la date $t$, notée $\pi_{t}$, est la somme des variations (en $\%$ ) de prix individuels $p_{i}$ observés entre deux dates $t$ et $t-1$ (Klenow et Kryvtsov, 2008) :

$$
\pi_{t}=\frac{1}{N} \sum_{i=1}^{N} \frac{p_{i, t}-p_{i, t-1}}{p_{i, t-1}}
$$

En introduisant une indicatrice de changement de prix, l'inflation se décompose en une marge extensive et une marge intensive :

$$
\pi_{t}=\left(\frac{1}{N} \sum_{i=1}^{N} I_{i, t}\right) \times\left(\frac{\sum_{i=1}^{N}\left(\frac{p_{i, t}-p_{i, t-1}}{p_{i, t-1}}\right)}{\sum_{i=1}^{N} I_{i, t}}\right)=f_{t} \times d p_{t}
$$

avec $I_{i t}=1$ si $p_{i t} \neq p_{i t-1}$ et $I_{i t}=0$ si $p_{i t}=p_{i t-1}$. L'inflation est alors le produit de la fréquence de changements de prix $f_{t}$ et la taille moyenne des changements de prix (en excluant les variations de prix égales à 0 ) à la date $t d p_{t}$. Wulfsberg (2010) montre que la moyenne des changements de prix $d p_{t}$ est une mesure biaisée de la marge intensive parce qu'elle est 
influencée par la marge extensive et suggère une décomposition de l'inflation entre hausses et baisses de prix :

$$
\pi_{t}=f_{t}^{+} \times d p_{t}^{+}+f_{t}^{-} \times d p_{t}^{-}
$$

où $f_{t}^{+}$et $f_{t}^{-}$sont les fréquences de hausses et de baisses (i.e. la marge extensive) alors que $d p_{t}^{+}$et $d p_{t}^{-}$sont les tailles moyennes de hausses et de baisses (i.e. la marge intensive).

Nous menons ensuite cette décomposition sur les données de fréquences et de tailles moyennes calculées par jour sur la période de l'échantillon. Les corrélations entre les différents indicateurs de rigidité des prix et l'inflation sont présentées dans le tableau 6. Les corrélations les plus fortes sont obtenues entre l'inflation et les fréquences de hausses et de baisses. Les coefficients estimés sont supérieurs à 0,8 . La fréquence des baisses de prix (resp. de hausses) diminue (resp. augmente) avec l'inflation. Enfin, les corrélations avec les tailles moyennes de prix sont plus faibles mais significatives. En particulier pour les supermarchés, les corrélations entre inflation et taille de prix sont positives : quand l'inflation augmente, la taille moyenne des hausses (resp. baisses) augmente (resp. diminue). Les résultats obtenus pour les autres distributeurs sont plus ambigus et parfois non-significatifs. Au total, il apparaît alors que les variations d'inflation sont principalement dues aux variations temporelles de la fréquence des hausses et des baisses de prix. Le modèle de coût d'ajustement est le modèle qui reproduit le mieux cette corrélation (Klenow et Kryvstov, 2008).

Tableau 6 : Corrélations entre inflation, fréquences et tailles des changements de prix (fréquence quotidienne, janvier 2007-juin 2009)

\begin{tabular}{|c|c|c|c|c|c|}
\hline & & \multicolumn{4}{|c|}{$\begin{array}{l}\text { Corrélation avec } \pi \\
\text { (p-value) }\end{array}$} \\
\hline & & $f+$ & $f-$ & $d p+$ & $|d p-|$ \\
\hline \multicolumn{6}{|l|}{ Diesel } \\
\hline & & 0,84 & $-0,84$ & 0,09 & $-0,37$ \\
\hline & Ensemble & $(0,00)$ & $(0,00)$ & $(0,02)$ & $(0,00)$ \\
\hline & C.urato & 0,83 & $-0,84$ & 0,28 & $-0,47$ \\
\hline & Supermarches & 082 & -080 & - 050 & -013 \\
\hline & Autres distributeurs & $(0,00)$ & $(0,00)$ & $(0,00)$ & $(0,00)$ \\
\hline \multicolumn{6}{|l|}{ SP 95} \\
\hline & & 0,82 & $-0,83$ & 0,06 & $-0,34$ \\
\hline & Ensemble & $(0,00)$ & $(0,00)$ & $(0,10)$ & $(0,00)$ \\
\hline & & 0,81 & $-0,84$ & 0,24 & $-0,47$ \\
\hline & Supermarchés & $(0,00)$ & $(0,00)$ & $(0,00)$ & $(0,00)$ \\
\hline & & 0,80 & $-0,79$ & $-0,21$ & $-0,05$ \\
\hline & Autres distributeurs & $(0,00)$ & $(0,00)$ & $(0,00)$ & $(0,13)$ \\
\hline
\end{tabular}

Lecture : $f$ désigne la fréquence quotidienne de changement de prix (c'est-à-dire le nombre de changements de prix observés chaque jour de l'échantillon sur le nombre total d'observations par jour), $d p$ la taille moyenne de changement de prix (parmi 
les stations qui ont changé leur prix) par date. Les signes + (resp. -) en exposant désignent les hausses (resp. les baisses) de prix. La journée du dimanche a été exclue.

Source: Données individuelles quotidiennes issues du site internet www.prix-carburants.gouv.fr (ensemble des stationsservice disponibles, janvier 2007-juin 2009), calculs des auteurs.

Afin d'illustrer les contributions respectives de la marge intensive ou extensive, nous recomposons des inflations «contrefactuelles » en faisant l'hypothèse que les fréquences ou les tailles de changement sont constantes au cours du temps (Wulfsberg, 2010). Pour évaluer la variabilité de l'inflation qui n'est due qu'aux variations de la fréquence des hausses et des baisses (i.e. la marge extensive), nous recomposons l'inflation suivante :

$$
\pi_{t}^{*} \mid f=f_{t}^{+} \times \overline{d p^{+}}+f_{t}^{-} \times \overline{d p^{-}}
$$

où $\overline{d p^{+}}$(resp. $\overline{d p^{-}}$) est la moyenne de la taille des hausses (resp. des baisses) de prix et $\pi_{t}^{*} \mid f$ l'inflation sous hypothèse que la taille des hausses et des baisses à chaque date est égale à sa moyenne. $\pi_{t}^{*} \mid f$ est donc l'inflation sous hypothèse que les changements de prix sont endogènes et que l'inflation varie avec la fréquence des changements. De même, nous calculons $\pi_{t}^{*} \mid d p$, l'inflation sous hypothèse que la fréquence des changements est constante à sa valeur moyenne, et que l'inflation varie uniquement avec la taille des changements :

$$
\pi_{t}^{*} \mid d p=\overline{f^{+}} \times d p_{t}^{+}+\overline{f^{-}} \times d p^{-}
$$

où $\overline{f^{+}}$(resp. $\overline{f^{-}}$) est la moyenne de la fréquence des hausses (resp. des baisses) de prix.

Le graphique 7 présente ces inflations recomposées ${ }^{\mathrm{xxi}}$. Les fréquences de hausses et de baisses contribuent le plus fortement aux variations de l'inflation des prix des carburants : les inflations recomposées en supposant que seules les fréquences varient sont les plus corrélées à l'inflation. La taille des changements de prix a une contribution très faible à l'inflation. Les variations d'inflation sont donc principalement expliquées par la marge extensive. Enfin, même sur la période 2008-2009 où la volatilité des chocs est grande, c'est toujours la marge extensive qui contribue le plus aux variations de l'inflation des prix des carburants. 


\section{Graphique 7 : Recomposition de l'inflation hebdomadaire des prix du diesel et du SP 95} (en \%)

Graphique 7.1 : Diesel

$$
7.1 .1 \pi_{t}^{*} \mid f
$$

$7.1 .2 \pi_{t}^{*} \mid d p$
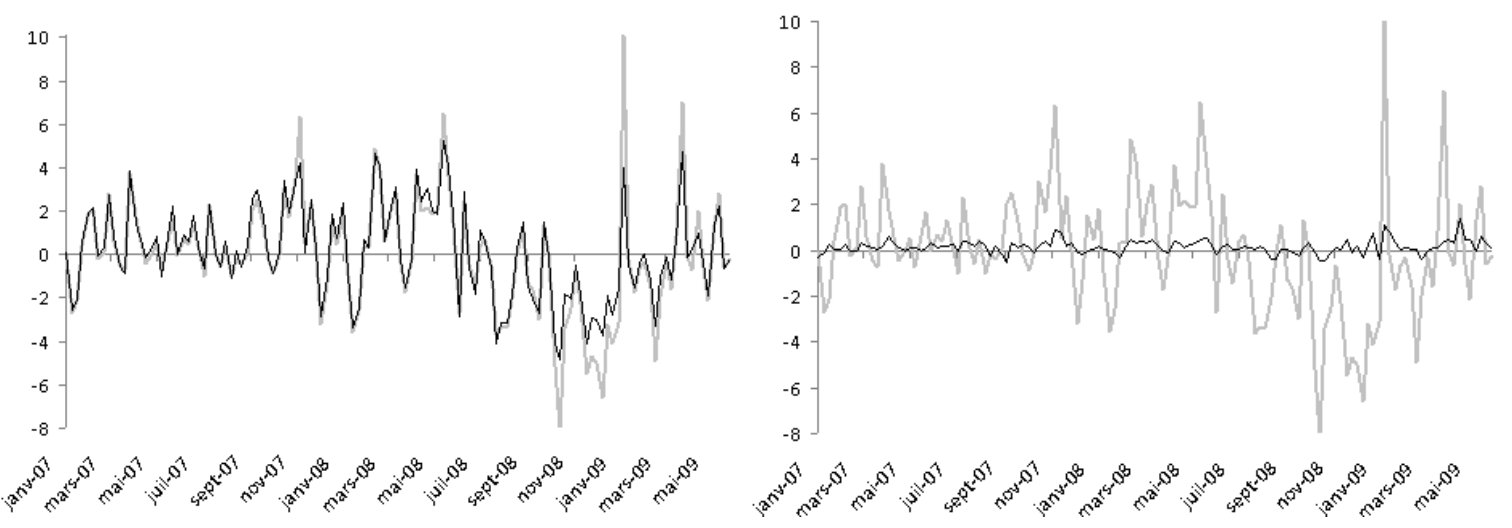

Graphique 7.2 : SP 95

$$
7.2 .1 \pi_{t}^{*} \mid f
$$

$7.2 .2 \pi_{t}^{*} \mid d p$
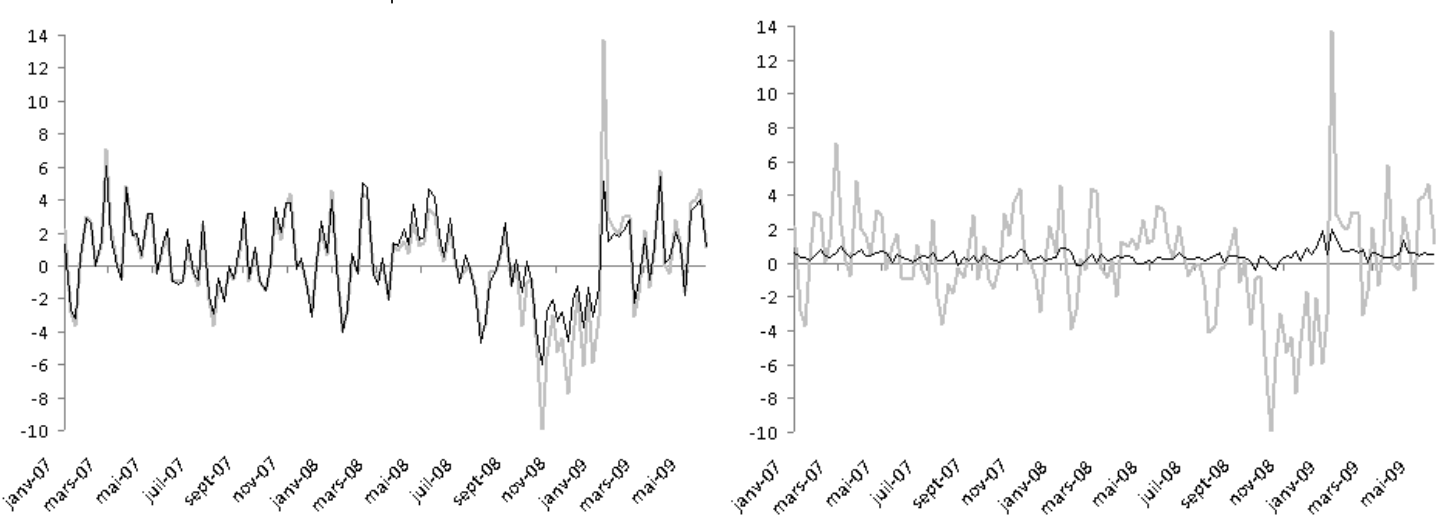

Lecture : En gris continu, l'inflation hebdomadaire calculée comme le cumul de l'inflation quotidienne recomposée à l'aide de la formule : $\pi_{t}=f_{t}^{+} \times d p_{t}^{+}+f_{t}^{-} \times d p_{t}^{-}$. En noir pointillé, l'inflation hebdomadaire recomposée selon différentes hypothèses sur la fréquence et la taille des changements ; $\pi_{t}^{*} \mid f$ inflation sous hypothèse que la taille moyenne des changements est constante, égale à sa moyenne $; \pi_{t}^{*} \mid d p$ inflation sous hypothèse que la fréquence des changements est constante, égale à sa moyenne.

Source: Données individuelles quotidiennes issues du site internet www.prix-carburants.gouv.fr (ensemble des stationsservice disponibles, janvier 2007-juin 2009), calculs des auteurs.

\section{Variations temporelles de la fréquence de changement de prix}

Le modèle de coût d'ajustement suppose que la décision de changer de prix est endogène et dépend de l'environnement économique. Nous analysons maintenant les déterminants des variations dans le temps de la fréquence des changements de prix. Nous régressons pour cela les fréquences quotidiennes moyennes de hausses et de baisses de prix sur l'évolution du cours du prix du diesel ou du SP 95 cotés à Rotterdam (en euros), approximant le coût marginal. Le modèle estimé est le suivant :

$$
f_{t}=\sum_{i=0}^{j} \alpha_{i} \Delta p_{t-i-6, t-i}^{\text {Rotterdam }}+\beta x_{t}+\varepsilon_{t}
$$


où $t$ est un indice quotidien, $f_{t}$ est la fréquence moyenne par jour de hausses ou de baisses de prix, $\Delta p_{t-i-6, t-i}^{\text {Rotterdam }}$ est le taux de croissance hebdomadaire (calculé sur la semaine précédant le jour $t$, c'est-à-dire entre le jour $t-6$ et le jour $t$ (le dimanche étant exclu); nous considérons ensuite les retards de cette variable par exemple l'évolution entre les jours $t-12$ et $t-6, i=0,6$, $12, \ldots, j)$ du prix du produit raffiné (diesel ou SP 95) à Rotterdam converti en euros, $x_{t}$ est la variabilité des prix Rotterdam sur les deux semaines précédant le jour $t$ et des variables indicatrices de jour de la semaine et $\varepsilon_{t}$ est un résidu normal centré. Nous avons retenu quatre semaines de retard maximum pour le taux de croissance du prix du produit raffiné à Rotterdam.

Les résultats de ces régressions pour le prix du diesel et du SP 95 sont présentés dans le tableau 7. Une hausse du prix d'1\% du produit raffiné à Rotterdam un jour donné augmenterait la fréquence quotidienne de hausses de prix et diminuerait la fréquence de baisses d'un peu plus de 2 pp pour le diesel et d'un peu moins de 1,5 pp pour le SP 95. L'effet des variations du prix «Rotterdam » est graduel : la durée de la transmission d'un choc est estimée entre 2 et 3 semaines pour le SP 95 et le diesel pour les hausses et les baisses de prix $^{\text {xxii }}$. Ces résultats confirment deux observations : (i) les prix sont rigides : les stationsservice changent leur prix avec retard par rapport aux variations de coût marginal ; (ii) la décision de changement de prix est endogène, elle dépend de l'environnement économique. Enfin, à partir des résultats du tableau 7 et de la décomposition de l'inflation en marge extensive et marge intensive, nous calculons dans quelle mesure, suite à une variation du prix Rotterdam de $1 \%$ un jour donné, les fréquences des hausses et des baisses de prix contribuent à la variation de l'inflation des prix des carburants. Un choc de $1 \%$ sur le prix « Rotterdam » un jour donné a pour effet d'augmenter la fréquence de hausses et diminuer la fréquence de baisses et augmente ainsi l'inflation de $0,77 \mathrm{pp}$ pour le diesel et $0,61 \mathrm{pp}$ pour le SP 95 (en cumulé sur trois semaines) $)^{\text {xxiii }}$. 
Tableau 7 : Déterminants de la fréquence de changement de prix des carburants

\begin{tabular}{|c|c|c|c|c|}
\hline & \multicolumn{2}{|c|}{ Diesel } & \multicolumn{2}{|c|}{ Sans plomb 95} \\
\hline & $f+$ & $f-$ & $f+$ & $f-$ \\
\hline$\Delta p_{t-6, t}^{\text {Rotterdam }}$ & $\begin{array}{c}1,147 * * * \\
(0,123)\end{array}$ & $\begin{array}{c}-1,116^{* * *} \\
(0,070)\end{array}$ & $\begin{array}{c}0,872 * * * \\
(0,063)\end{array}$ & $\begin{array}{c}-0,833 * * * \\
(0,060)\end{array}$ \\
\hline$\Delta p_{t-12, t-6}^{\text {Rotterdam }}$ & $\begin{array}{l}0,769 * * * \\
(0,129)\end{array}$ & $\begin{array}{c}-0,761 * * * \\
(0,072)\end{array}$ & $\begin{array}{l}0,439 * * * \\
(0,084)\end{array}$ & $\begin{array}{c}-0,453 * * * \\
(0,052)\end{array}$ \\
\hline$\Delta p_{t-18, t-12}^{\text {Rotterdam }}$ & $\begin{array}{c}0,150^{*} \\
(0,088)\end{array}$ & $\begin{array}{l}-0,160 * * * \\
\quad(0,059)\end{array}$ & $\begin{array}{l}0,061 \\
(0,051)\end{array}$ & $\begin{array}{c}-0,146^{* * * *} \\
\quad(0,044)\end{array}$ \\
\hline$\Delta p_{t-24, t-18}^{\text {Rotterdam }}$ & $\begin{array}{l}0,119 \\
(0,086)\end{array}$ & $\begin{array}{l}0,088 \\
(0,067)\end{array}$ & $\begin{array}{l}0,091 \\
(0,059)\end{array}$ & $\begin{array}{l}-0,018 \\
(0,049)\end{array}$ \\
\hline Lundi & $\begin{array}{c}-2,037 * * \\
(0,952)\end{array}$ & $\begin{array}{c}-7,759 * * * \\
(0,843)\end{array}$ & $\begin{array}{c}-2,437 * * * \\
(0,893)\end{array}$ & $\begin{array}{c}-7,016 * * * \\
(0,801)\end{array}$ \\
\hline Mardi & $\begin{array}{l}1,706 \\
(1,141)\end{array}$ & $\begin{array}{c}-4,321 * * * \\
(0,899)\end{array}$ & $\begin{array}{l}1,705 \\
(1,096)\end{array}$ & $\begin{array}{c}-4,134 * * * \\
(0,865)\end{array}$ \\
\hline Mercredi & $\begin{array}{l}-0,057 \\
(0.985)\end{array}$ & $\begin{array}{l}-4,577 * * * \\
\quad(0,846)\end{array}$ & $\begin{array}{l}-0,228 \\
(0,959)\end{array}$ & $\begin{array}{l}-4,389 * * * \\
\quad(0,818)\end{array}$ \\
\hline Jeudi & $\begin{array}{c}1,866^{* *} \\
(0,769)\end{array}$ & $\begin{array}{l}-3,541 * * * \\
(0,712)\end{array}$ & $\begin{array}{c}-1,759 * * \\
(0,762)\end{array}$ & $\begin{array}{l}-3,502 * * * \\
(0,683)\end{array}$ \\
\hline Vendredi & Ref. & Ref. & Ref. & Ref. \\
\hline Samedi & $\begin{array}{c}-4.148 * * * \\
(0,732) \\
\end{array}$ & $\begin{array}{c}-6,658 * * * \\
(0,680) \\
\end{array}$ & $\begin{array}{c}-4,474 * * * \\
(0,716) \\
\end{array}$ & $\begin{array}{c}-6,215^{* * *} \\
(0,638) \\
\end{array}$ \\
\hline $\begin{array}{l}\text { Coefficient de variation des prix } \\
\text { sur } 10 \text { jours }\end{array}$ & $\begin{array}{c}1,200 * * * \\
(0,367)\end{array}$ & $\begin{array}{c}1,649 * * * \\
(0,218)\end{array}$ & $\begin{array}{c}0,646^{* *} \\
(0,258)\end{array}$ & $\begin{array}{c}1,025^{* * *} * \\
(0,166)\end{array}$ \\
\hline Constante & $\begin{array}{c}8,746 * * * \\
(1,126)\end{array}$ & $\begin{array}{c}10,824 * * * \\
(0,901)\end{array}$ & $\begin{array}{c}9,341 * * * \\
(1,187) \\
\end{array}$ & $\begin{array}{c}10,645 * * * \\
(0,872)\end{array}$ \\
\hline Nombre d'observations & 732 & 732 & 732 & 732 \\
\hline
\end{tabular}

Lecture : Les colonnes contiennent les résultats des estimations par moindres carrés ordinaires. Les variables endogènes sont la fréquence de baisse $(f-)$, et la fréquence de hausse $(f+)$ de prix (calculées en $\%$ ), par jour (dimanches exclus). Ces statistiques sont calculées pour l'ensemble des stations-service. L'évolution du prix de marché « Rotterdam » $\Delta p_{t-6, t}^{\text {Rotterdam }}$ est calculée sur une semaine précédant le jour t considéré. $\Delta p_{t-12, t-6}^{\text {Rotterdam }}, \Delta p_{t-18, t-12}^{\text {Rotterdam }}$ et $\Delta p_{t-24, t-18}^{\text {Rotterdam }}$ est l'évolution du prix Rotterdam retardé de deux, trois ou quatre semaines. Le coefficient de variation des prix sur 2 semaines est l'écart-type du prix de marché sur les 10 jours ouvrables précédents sur le prix moyen constaté. Les estimations sont corrigées de l'autocorrélation à l'aide de la matrice de Newey-West. Niveau de significativité : *** $1 \%$, ** $5 \%$, * $10 \%$.

Source: Données individuelles quotidiennes issues du site internet www.prix-carburants.gouv.fr (ensemble des stationsservice disponibles) et U.S. Energy Information Administration, calculs des auteurs.

Toutefois, les effets des jours de la semaine sont aussi significatifs, ce qui suggère que la décision de changement de prix est aussi influencée par d'autres éléments que le coût marginal et est donc en partie prédéterminée. Le tableau 8 présente les fréquences de changements de prix par jour de la semaine pour le diesel et le SP 95. Les stations-service changent en moyenne plus souvent leur prix le mardi et le vendredi : la fréquence de changements de prix est alors comprise entre 24 et $28 \%$ pour le diesel ou le SP 95. La fréquence est plus faible le mercredi et jeudi autour de 21-23\%. Les changements de prix sont plus rares le samedi et le lundi où la fréquence de changements de prix se situe entre 15 et $16 \%$. Le dimanche, très peu de stations-service modifient leurs prix ${ }^{\mathrm{xxiv}}$. Enfin, les pics au cours de la semaine ne sont pas les mêmes pour les hausses et les baisses : les baisses sont 
significativement plus fréquentes le vendredi (en particulier dans les supermarchés, Tableau A1 en annexe) alors que les hausses sont plus fréquentes le mardi et le vendredi. Si on relie cette saisonnalité aux fonctions de hasard présentées précédemment, il semble que les stations-service changent leurs prix une fois par semaine, le même jour. Comme le montrent Nakamura et Steinsson (2011), en présence d'habitudes de consommation et d'asymétrie d'information, les détaillants ont intérêt à éviter de changer leur prix fréquemment, communiquer sur une politique de prix bas pourrait alors crédibiliser l'existence de contrats implicites de prix fixes. Dans ce cas, il peut être intéressant pour les stations de concentrer leurs baisses de prix en fin de semaine au moment où la demande est plus forte.

Tableau 8 : Fréquence des changements de prix en fonction des jours de la semaine (\%)

\begin{tabular}{cccccc|ccccc}
\hline \hline & \multicolumn{9}{c}{ Diesel } \\
& $f$ & $f+$ & $f-$ & $d p+$ & $d p-$ & $f$ & $f+$ & $f-$ & $d p+$ & $d p-$ \\
\hline Lundi & 16,47 & 9,14 & 7,33 & 2,42 & $-2,46$ & 15,45 & 8,94 & 6,51 & 3,04 & $-3,02$ \\
Mardi & 24,98 & 13,63 & 11,34 & 2,22 & $-2,29$ & 23,55 & 13,68 & 9,87 & 2,73 & $-2,76$ \\
Mercredi & 22,93 & 11,94 & 10,98 & 2,37 & $-2,26$ & 21,28 & 11,81 & 9,47 & 2,91 & $-2,76$ \\
Jeudi & 22,14 & 10,21 & 11,92 & 2,34 & $-2,28$ & 20,64 & 10,22 & 10,42 & 2,81 & $-2,80$ \\
Vendredi & 27,66 & 12,01 & 15,65 & 2,26 & $-2,57$ & 26,16 & 12,09 & 14,07 & 2,77 & $-3,10$ \\
Samedi & 16,57 & 7,81 & 8,77 & 2,35 & $-2,47$ & 15,28 & 7,59 & 7,69 & 2,88 & $-3,04$ \\
Dimanche & 1,06 & 0,60 & 0,46 & 3,27 & $-2,88$ & 0,99 & 0,58 & 0,41 & 4,05 & $-3,48$ \\
\hline \hline
\end{tabular}

Lecture : $f$ désigne la fréquence moyenne de changement de prix par jour de la semaine, $d p$ la taille moyenne de changement de prix (parmi les stations ayant changé leur prix). Les signes + (resp. -) en exposant désignent les hausses (resp. les baisses) de prix. En moyenne, chaque lundi, 16,5\% des prix du diesel sont modifiés.

Source: Données individuelles quotidiennes issues du site internet www.prix-carburants.gouv.fr (ensemble des stationsservice disponibles), calculs des auteurs.

\section{Variations temporelles de la taille des changements de prix}

Enfin, nous étudions les déterminants des variations temporelles de la taille moyenne des changements de prix (parmi les stations ayant modifié leur prix) même si elles sont faibles et contribuent peu aux variations de l'inflation. Comme pour les fréquences, nous étudions la relation entre la taille moyenne des changements de prix HT (en excluant les variations de prix nulles) et les variations de coût marginal approximées par les évolutions du prix des produits raffinés coté à Rotterdam. Le modèle estimé est le suivant :

$$
d p_{t}=\sum_{i=0}^{j} \gamma_{i} \Delta p_{t-6-i, t-i}^{\text {Rotterdam }}+\phi z_{t}+v_{t}
$$

où $d p_{t}$ est la taille moyenne de hausses ou de baisses de prix par jour, $\Delta p_{t-i-6, t-i}^{\text {Rotterdam }}$ est le taux de croissance hebdomadaire (calculé sur la semaine précédant le jour $t$, c'est-à-dire entre le jour $t-6$ et le jour $t$; nous considérons ensuite les retards de cette variable par exemple l'évolution entre les jours $t-12$ et $t-6, i=0,6,12, \ldots, j$ ) du prix du produit raffiné (diesel ou SP 95) à Rotterdam converti en euros, $z_{t}$ est la variabilité des prix Rotterdam sur les deux 
semaines précédant la date $t$, des variables indicatrices de jour de la semaine et la fréquence des hausses ou des baisses de prix à la date $t$, et $v_{t}$ est un résidu normal centré.

Les résultats de ces régressions sont présentés dans le tableau $9^{\mathrm{xxv}}$. L'effet des évolutions du prix Rotterdam est faible ou non-significatif sur les tailles moyennes des changements de prix. L'effet le plus fort est celui associé à l'évolution du prix Rotterdam entre 6 et 12 jours avant la date $t$, toutefois cet effet est très limité : une hausse ou une baisse de $1 \%$ du prix Rotterdam aurait un effet compris entre 0,02 et 0,04 pp sur la taille des baisses ou des hausses un jour donnéx ${ }^{x v i}$. Au total, en reprenant la décomposition de l'inflation entre marge intensive et extensive et les résultats du tableau 9, la contribution des tailles de hausses et de baisses à la variation de l'inflation est faible: après un choc de $1 \%$ sur le prix «Rotterdam» un jour donné, les variations de taille moyenne contribuent à augmenter l'inflation de 0,02 pp pour le diesel et 0,01 pp pour le SP95 (en cumulé sur 3 semaines). Ce résultat est cohérent avec la faible contribution de la marge intensive à l'inflation : quand le prix du produit raffiné à Rotterdam augmente, les hausses de prix dans les stations sont plus fréquentes (et le nombre de baisses diminue), ce qui contribue à augmenter l'inflation mais les tailles moyennes de hausses ou de baisses restent presque inchangées. Par ailleurs, les différences entre les jours de la semaine sont relativement faibles: les hausses sont en moyenne plus fortes le lundi et le samedi alors que les baisses sont en moyenne un peu plus importantes le vendredi. En fin de semaine, les baisses sont donc plus fréquentes et de plus grande ampleur : les stations ont davantage intérêt à modifier leur prix la veille des week-ends en prévision de la hausse de demande, elles peuvent diminuer leurs marges en menant des opérations dites «prix-coûtant», attirer plus de consommateurs et crédibiliser leur engagement sur des prix peu variables. Enfin, la variance des chocs de prix des produits Rotterdam a tendance à augmenter significativement l'ampleur des changements de prix prise en valeur absolue.

Tableau 9 : Déterminants de la taille des changements de prix hors taxes

\begin{tabular}{|c|c|c|c|c|}
\hline & \multicolumn{2}{|c|}{ Diesel } & \multicolumn{2}{|c|}{ SP 95} \\
\hline & $d p+$ & $|d p-|$ & $d p+$ & $|d p-|$ \\
\hline$\Delta p_{t-6, t}^{\text {Rotterdam }}$ & $\begin{array}{l}-0,005 \\
(0,007)\end{array}$ & $\begin{array}{c}0,008 \\
(0,007)\end{array}$ & $\begin{array}{l}-0,006 \\
(0,012)\end{array}$ & $\begin{array}{c}0,002 \\
(0,008)\end{array}$ \\
\hline$\Delta p_{t-12, t-6}^{\text {Rotterdam }}$ & $\begin{array}{c}0,024 * * \\
(0,011)\end{array}$ & $\begin{array}{c}-0,027 * * * \\
(0,006)\end{array}$ & $\begin{array}{l}-0,001 \\
(0,008)\end{array}$ & $\begin{array}{c}-0,035^{* * *} * \\
(0,005)\end{array}$ \\
\hline$\Delta p_{t-18, t-12}^{\text {Rotterdam }}$ & $\begin{array}{l}-0,007 \\
(0,008)\end{array}$ & $\begin{array}{c}-0,018 * * \\
(0,008)\end{array}$ & $\begin{array}{l}-0,006 \\
(0,008)\end{array}$ & $\begin{array}{l}-0,009 \\
(0,006)\end{array}$ \\
\hline$\Delta p_{t-24, t-18}^{\text {Rotterdam }}$ & $\begin{array}{c}0,017 \\
(0,014) \\
\end{array}$ & $\begin{array}{c}0,001 \\
(0,008) \\
\end{array}$ & $\begin{array}{c}0,008 \\
(0,010) \\
\end{array}$ & $\begin{array}{c}-0,001 \\
(0,006) \\
\end{array}$ \\
\hline Lundi & $\begin{array}{c}0,360 * * * \\
(0,076)\end{array}$ & $\begin{array}{l}-0,061 \\
(0,055)\end{array}$ & $\begin{array}{c}0,557 * * * \\
(0,092)\end{array}$ & $\begin{array}{c}-0,043 \\
(0,069)\end{array}$ \\
\hline Mardi & $\begin{array}{c}-0,055 \\
(0,062)\end{array}$ & $\begin{array}{c}-0,203 * * * \\
(0,057)\end{array}$ & $\begin{array}{c}0,006 \\
(0,075)\end{array}$ & $\begin{array}{c}-0,231 * * * \\
(0,066)\end{array}$ \\
\hline Mercredi & $\begin{array}{l}-0,040 \\
(0,052)\end{array}$ & $\begin{array}{c}-0,200 * * * \\
(0,056)\end{array}$ & $\begin{array}{c}0,002 \\
(0,069)\end{array}$ & $\begin{array}{c}-0,255^{* * *} \\
(0,067)\end{array}$ \\
\hline Jeudi & $\begin{array}{l}-0,023 \\
(0,042)\end{array}$ & $\begin{array}{c}-0,119 * * \\
(0,057)\end{array}$ & $\begin{array}{c}-0,044 \\
(0,055)\end{array}$ & $\begin{array}{c}-0,120^{*} \\
(0,067)\end{array}$ \\
\hline Vendredi & Ref & Ref & Ref & Ref \\
\hline
\end{tabular}




\begin{tabular}{lcccc} 
Samedi & $0,329 * * *$ & 0,026 & $0,414 * * *$ & 0,003 \\
& $(0,067)$ & $(0,043)$ & $(0,081)$ & $(0,055)$ \\
\hline \multirow{2}{*}{ Fréquence des changements } & 0,005 & $0,015 * * *$ & $0,009 *$ & $0,016 * *$ \\
\multirow{2}{*}{ Coefficient de variation des prix } & $(0,004)$ & $(0,005)$ & $(0,005)$ & $(0,006)$ \\
sur 10 jours & $0,332 * * *$ & $0,227 * * *$ & $0,342 * * *$ & $0,261 * * *$ \\
\hline \multirow{2}{*}{ Constante } & $(0,032)$ & $(0,018)$ & $(0,032)$ & $(0,017)$ \\
\hline Nombre d'observations & $1,179 * * *$ & $1,389 * * *$ & $1,303 * * *$ & $1,555 * * *$ \\
\hline
\end{tabular}

Lecture : Les colonnes contiennent les résultats des estimations par moindres carrés ordinaire. Les variables endogènes sont la taille moyenne des baisses $(d p$-), et la taille moyenne des hausses $(d p+)(\mathrm{en} \%)$, par jour, les dimanches ont été exclus. Ces statistiques sont calculées pour l'ensemble des stations-service. L'évolution du prix de marché « Rotterdam » $\Delta p_{t-6, t}^{\text {Rotterdam }}$ est calculée sur une semaine précédant le jour t considéré. $\Delta p_{t-12, t-6}^{\text {Rotterdam }}, \Delta p_{t-18, t-12}^{\text {Rotterdam }}$ et $\Delta p_{t-24, t-18}^{\text {Rotterdam }}$ est l'évolution du prix Rotterdam retardé de deux, trois, quatre semaines. Le coefficient de variation des prix sur 2 semaines est l'écart-type du prix de marché sur les 10 jours ouvrables précédents sur le prix moyen constaté. Les estimations sont corrigées de l'autocorrélation à l'aide de la matrice de Newey-West. Niveau de significativité : *** $1 \%$, ** $5 \%$, * $10 \%$.

Source: Données individuelles quotidiennes issues du site internet www.prix-carburants.gouv.fr (ensemble des stationsservice disponibles) et U.S. Energy Information Administration, calculs des auteurs.

\section{Conclusion}

Contrairement aux prix du pétrole ou aux prix de gros des carburants, les prix des carburants distribués dans les stations-service ne changent pas quotidiennement mais en moyenne une fois par semaine. Ils seraient donc rigides. Les stations-service présentes dans les supermarchés ont tendance à modifier leur prix plus fréquemment que les autres types de distributeurs. Les prix se terminant par un « $0 »$ ou un «9», dits psychologiques ont toutes choses égales par ailleurs, des durées plus longues. La distribution des changements de prix présente des particularités : les petits changements de prix (compris entre $-2 \%$ et $+2 \%$ ) sont plutôt rares. Enfin, il existe une corrélation positive entre taille des changements et durée des prix entre deux changements. Les variations temporelles des prix des carburants sont principalement expliquées par des variations des fréquences de hausses et de baisses de prix. Ces variations sont dues à des changements plus fréquents certains jours de la semaine (mardi et vendredi) et par les variations des cours des produits raffinés à Rotterdam.

Ces faits stylisés peuvent permettre d'invalider certains modèles de rigidité des prix. Les modèles de dépendance au temps ne peuvent pas reproduire la distribution bimodale des tailles de changements de prix observés ou la corrélation positive entre la durée et la taille des changements. Les modèles de coût d'ajustement (Golosov et Lucas, 2007) sont davantage capables de reproduire ces deux phénomènes ainsi que l'effet significatif du coût marginal sur la probabilité de changement de prix. Toutefois, ces modèles ne peuvent pas expliquer pourquoi les prix sont modifiés à des dates régulières dans la semaine. Les modèles associant coût d'ajustement et rigidité informationnelle (Woodford, 2009; Alvarez et al., 2011) apparaissent comme les plus prometteurs dans la mesure où ils permettent d'expliquer (i) que les stations-service ne révisent pas leur ensemble d'information tous les jours mais de façon régulière, certains jours dans la semaine (en particulier, en présence de contrats implicites entre la station-service et les consommateurs) ; (ii) quand elles révisent leur prix, leur décision de changer de prix dépend des variations de coût marginal; (iii) que le coût d'ajustement 
(possiblement faible pour les stations-service) sera relativement plus important si elles adoptent un prix dit «psychologique ».

Cette étude présente des faits stylisés sur la fixation des prix des carburants et met en évidence de façon indirecte la présence de rigidités de prix. Une piste de recherche ultérieure pourrait consister à mesurer et tester directement la nature et l'ampleur des coûts d'ajustement des prix dans les stations-service via par exemple des enquêtes (Blinder et al., 1998). 


\section{Bibliographie}

Alvarez F., Lippi F. et Paciello L. (2011). "Optimal Price Setting with Observation and Menu Costs”, Quarterly Journal of Economics, vol. 126, n 4, pp. 1909-1960.

Asplund M., Eriksson R. et Friberg R. (2000). "Price Adjustments by a Gasoline Retail Chain", Scandinavian Journal of Economics, vol. 102, n ${ }^{\circ}$, pp. 101-21.

Audenis C., Biscourp P. et Riedinger N. (2002). "Le prix des carburants est plus sensible à une hausse qu'à une baisse du brut", Économie et Statistique, n 359-360, pp. 149-165.

Baudry L., Le Bihan H., Sevestre P. et Tarrieu S. (2005). "La rigidité des prix en France : quelques enseignements des relevés de prix à la consommation", Économie et Statistique, $\mathrm{n}^{\circ}$ 386, pp. 37-57.

Bellec G., Bolliet A., Meslin O., Ravignon B et Serres H. (2012). "Les prix, les marges et la consommation de carburants", Rapport de l'Inspection générale des finances n 2012-M-06504 et du Conseil général de l'économie, de l'industrie, de l'énergie et des technologies $\mathrm{n}^{\circ}$ 2012/22/CGEIET/SG.

Berardi N., Gautier E. et Le Bihan H. (2013). "Les ajustements individuels de prix à la consommation en France : de nouveaux résultats sur la période 2003-2011", Économie et Statistique, $\mathrm{n}^{\circ}$ 460-461, pp. 5-35.

Bils M. et Klenow P.J. (2004). "Some Evidence on the Importance of Sticky Prices", Journal of Political Economy, vol. 112, n 5, pp. 947-985.

Blinder A.S., Canetti E.R.D., Lebow D.E. et Rudd J.B. (1998). Asking About Prices: A New Approach to Understanding Price Stickiness, Éditions Russell Sage Foundation, 336 pages.

Calvet L. et Marical F. (2012). "Consommation de carburant : effets des prix à court et à long terme par type de population", Économie et Statistique, n 446, pp. 25-44.

Calvo G. (1983). "Staggered Prices in a Utility-Maximizing Framework", Journal of Monetary Economics, vol. 12, n 3, pp. 383-398.

Cavallo A. et Rigobon R. (2011). "The Distribution of the Size of Price Changes", Working Paper $\mathrm{n}^{\circ}$ 16760, National Bureau of Economic Research, 39 pages.

Davis M.C. (2007). "The Dynamics of Daily Retail Gasoline Prices", Managerial and Decision Economics, vol. 28, n ${ }^{\circ}$ 7, pp. 713-722.

Davis M.C. et Hamilton J.D. (2004). "Why Are Prices Sticky? The Dynamics of Wholesale Gasoline Prices", Journal of Money, Credit and Banking, vol. 36, $\mathrm{n}^{\circ}$ 1, pp. 17-37.

Dhyne E., Alvarez L., Le Bihan H., Veronese G., Dias D., Hoffman J., Jonker N., Lunneman P., Rumler F., et Vilmunen J. (2006). "Price Changes in the Euro Area and the United States: Some Facts from Individual Consumer Price Data", Journal of Economic Perspectives, vol. 20, $\mathrm{n}^{\circ}$ 2, pp. 171-192.

Douglas C. et Herrera A.-M. (2010). "Why are Gasoline Prices Sticky? A Test of Alternative Models of Price Adjustment", Journal of Applied Econometrics, vol. 25, n 6, pp. 903-928.

Fabiani S., Druant M., Hernando I., Kwapil C., Landau B., Loupias C., Martins F., Matha T., Sabbatini R., Stahl H., et Stokman A. (2006). "What Firms' Surveys Tell Us about Price-Setting Behavior in the Euro Area", International Journal of Central Banking, vol. 2, n ${ }^{\circ}$, pp. 3-47.

Gautier E. (2008). "La dynamique des changements de prix de production : une analyse à partir des relevés de prix à la production", Économie et Statistique, n 407, pp. 3-26.

Gautier E. (2009). "Les ajustements microéconomiques des prix : une synthèse des modèles théoriques et résultats empiriques", Revue d'Économie Politique, vol. 119, n 3, pp. 323-372. 
Gautier E. et Le Saout R. (2012). "The Dynamics of Gasoline Prices: Evidence from Daily French Micro Data", Document de travail n 375, Banque de France, 62 pages.

Golosov M. et Lucas R.E. (2007). "Menu Costs and Phillips Curves", Journal of Political Economy, vol. 115, pp. 171-199.

Hosken D.S., McMillan R.S. et Taylor C.T. (2008). "Retail Gasoline Pricing: What do we know?", International Journal of Industrial Organization, vol. 26, pp. 1425-1436.

Kempf H. (2006). "Rigidités nominales: le difficile passage de l'empirique au théorique", Économie et Statistique, $\mathrm{n}^{\circ}$ 386, pp. 81-89.

Klenow P.J. et Kryvtsov O. (2008). "State Dependent or Time Dependent Pricing: Does It Matter for Recent US Inflation?", Quarterly Journal of Economics, vol. 123, n 3, pp. 863904.

Klenow P.J. et Malin B.A. (2010). "Microeconomic Evidence on Price-Setting" in Handbook of Monetary Economics, Friedman B.M. et Woodford M. (ed.), Elsevier, vol. 3, ch. 6, pp. 231-284.

Knotek E.S. II (2010). "The Roles of Price Points and Menu Costs in Price Rigidity", Research Working Paper $\mathrm{n}^{\circ}$ 10-18, Federal Reserve Bank of Kansas City, 39 pages.

Lamotte O., Porcher T., Schalck C. et Silvestre S. (2013). "Asymmetric Gasoline Price Responses in France", Applied Economics Letters, vol. 20, n 5, pp. 457-461.

Levy D., Lee D., Chen H., Kauffman R. et Bergen M. (2011). "Price Points and Price Rigidity", Review of Economics and Statistics, vol. 93, n 4, pp. 1417-1431.

Meyler A. (2010). "The Pass-Through of Oil Prices into Euro Area Consumer Liquid Fuel Prices in an Environment of High and Volatile Oil Prices", Energy Economics, vol. 31, n ${ }^{\circ} 6$, pp. 867-881.

Nakamura E. et Steinsson J. (2008). "Five Facts About Prices: A Reevalutaion Of Menu Cost Model", Quarterly Journal of Economics, vol. 123, n 4, pp. 1415-1464.

Nakamura E. et Steinsson N. (2011). "Price Setting in Forward-Looking Customer Markets", Journal of Monetary Economics, vol. 58, n 3, pp. 220-233.

Nakamura E. et Steinsson N. (2013). "Price Rigidity: Microeconomic Evidence and Macroeconomic Implications", Annual Review of Economics, vol. 5, pp. 133-163.

Rotemberg J. (2005). "Customer Anger at Price Increases, Changes in the Frequency of Price Adjustment and Monetary Policy", Journal of Monetary Economics, vol. 52, $\mathrm{n}^{\circ}$ 4, pp. 829-852.

Rotemberg J. et Saloner G. (1987). “The Relative Rigidity of Monopoly Pricing”, American Economic Review, vol. 77, n 5, pp. 917-26.

Taylor J.B. (1980). "Aggregate Dynamics and Staggered Contracts", Journal of Political Economy, vol. 88, ${ }^{\circ}$ 1, pp. 1-23.

Woodford M. (2009). "Information-Constrained State-Dependent Pricing", Journal of Monetary Economics, vol. 56, pp. 100-124.

Wulfsberg F. (2009). "Inflation and Price Adjustments: Evidence from Norwegian Consumer Price Data 1975-2004”, Working Paper n 2009/11, Norges Bank, 36 pages.

Zbaracki M.J., Ritson M., Levy D., Dutta S. et Bergen M. (2004). "Managerial and Customer Costs of Price Adjustment: Direct Evidence from Industrial Markets", The Review of Economics and Statistics, vol. 86, $\mathrm{n}^{\circ} 2$, pp. 514-533.

Zimmerman P. R. (2012). "The Competitive Impact of Hypermarket Retailers on Gasoline Prices", Journal of Law and Economics, vol. 55, $n^{\circ}$ 1, pp. 27-41. 


\section{Annexes}

Tableaux A1 : Déterminants de la fréquence de changement de prix des carburants par type de distributeurs

a) Tableau A1.1 : Supermarchés

\begin{tabular}{|c|c|c|c|c|}
\hline & \multicolumn{2}{|c|}{ Diesel } & \multicolumn{2}{|c|}{ Sans plomb 95} \\
\hline & $f+$ & $f-$ & $f+$ & $f-$ \\
\hline$\Delta p_{t-6, t}^{\text {Rotterdam }}$ & $\begin{array}{c}1,134 * * * \\
(0,116)\end{array}$ & $\begin{array}{c}-1,092 * * * \\
(0,071)\end{array}$ & $\begin{array}{c}0,825 * * * \\
(0,059)\end{array}$ & $\begin{array}{c}-0,763 * * * \\
(0,048)\end{array}$ \\
\hline$\Delta p_{t-12, t-6}^{\text {Rotterdam }}$ & $\begin{array}{c}0,676 * * * \\
(0,112)\end{array}$ & $\begin{array}{c}-0,699 * * * \\
(0,074)\end{array}$ & $\begin{array}{c}0,394 * * * \\
(0,071)\end{array}$ & $\begin{array}{c}-0,424 * * * \\
(0,047)\end{array}$ \\
\hline$\Delta p_{t-18, t-12}^{\text {Rotterdam }}$ & $\begin{array}{c}0,095 \\
(0,083)\end{array}$ & $\begin{array}{c}-0,113^{*} \\
(0,062)\end{array}$ & $\begin{array}{c}0,045 \\
(0,047)\end{array}$ & $\begin{array}{c}-0,129 * * * \\
(0,041)\end{array}$ \\
\hline$\Delta p_{t-24, t-18}^{\text {Rotterdam }}$ & $\begin{array}{c}0,059 \\
(0,082) \\
\end{array}$ & $\begin{array}{c}0,090 \\
(0,069) \\
\end{array}$ & $\begin{array}{c}0,041 \\
(0,054)\end{array}$ & $\begin{array}{l}-0,003 \\
(0,043) \\
\end{array}$ \\
\hline Lundi & $\begin{array}{c}-0,422 \\
(1,142)\end{array}$ & $\begin{array}{l}-9,589 * * * \\
(0,993)\end{array}$ & $\begin{array}{c}-0,821 \\
(1,050)\end{array}$ & $\begin{array}{l}-8,666^{* * *} \\
(0,892)\end{array}$ \\
\hline Mardi & $\begin{array}{c}1,182 \\
(1,189)\end{array}$ & $\begin{array}{c}-7,543 * * * \\
(1,034)\end{array}$ & $\begin{array}{c}1,300 \\
(1,123)\end{array}$ & $\begin{array}{c}-7,117 * * * \\
(0,940)\end{array}$ \\
\hline Mercredi & $\begin{array}{l}-1,521 \\
(1,028)\end{array}$ & $\begin{array}{c}-7,128^{* * * *} \\
(0,979)\end{array}$ & $\begin{array}{l}-1,146 \\
(0,955)\end{array}$ & $\begin{array}{c}-7,173^{* * * *} \\
(0,890)\end{array}$ \\
\hline Jeudi & $\begin{array}{c}-3,216^{* * *} \\
(0,888)\end{array}$ & $\begin{array}{c}-6,072 * * * \\
(0,887)\end{array}$ & $\begin{array}{c}-3,090 * * * \\
(0,826)\end{array}$ & $\begin{array}{c}-5,957 * * * \\
(0,821)\end{array}$ \\
\hline Vendredi & Ref & Ref & Ref & Ref \\
\hline Samedi & $\begin{array}{c}-5,464 * * * \\
(0,817) \\
\end{array}$ & $\begin{array}{c}-9,253 * * * \\
(0,773)\end{array}$ & $\begin{array}{c}-5,377 * * * \\
(0,753) \\
\end{array}$ & $\begin{array}{c}-8,853 * * * \\
(0,722)\end{array}$ \\
\hline $\begin{array}{l}\text { Coefficient de variation des prix } \\
\text { sur } 10 \text { jours }\end{array}$ & $\begin{array}{c}0,679 * * \\
(0,335)\end{array}$ & $\begin{array}{c}1,316^{* * * *} \\
(0,227)\end{array}$ & $\begin{array}{c}0,353 \\
(0,221) \\
\end{array}$ & $\begin{array}{c}0,731 * * * \\
(0,153)\end{array}$ \\
\hline Constante & $\begin{array}{c}11,913 * * * \\
(1,208)\end{array}$ & $\begin{array}{c}15,241 * * * \\
(1,092)\end{array}$ & $\begin{array}{c}11,722^{* * * *} \\
(1,181)\end{array}$ & $\begin{array}{c}14,879 * * * \\
(1,028)\end{array}$ \\
\hline Nombre d'observations & 732 & 732 & 732 & 732 \\
\hline
\end{tabular}

b) Tableau A1.2: Autres distributeurs

\begin{tabular}{lcccc}
\hline \hline & \multicolumn{2}{c}{ Diesel } & \multicolumn{2}{c}{ Sans plomb 95 } \\
& $f+$ & $f-$ & $f+$ & $f-$ \\
\hline \multirow{2}{*}{$\Delta p_{t-6, t}^{\text {Rotterdam }}$} & $1,147 * * *$ & $-1,123^{* * *}$ & $0,909 * * *$ & $-0,890^{* * *}$ \\
$\Delta p_{t-12, t-6}^{\text {Rotterdam }}$ & $(0,133)$ & $(0,076)$ & $(0,074)$ & $(0,078)$ \\
\multirow{2}{*}{$\Delta p_{t-18, t-12}^{\text {Rotterdam }}$} & $0,850 * * *$ & $-0,808 * * *$ & $0,473 * * *$ & $-0,471 * * *$ \\
\multirow{2}{*}{$\Delta p_{t-24, t-18}^{\text {Rotterdam }}$} & $(0,150)$ & $(0,077)$ & $(0,100)$ & $(0,063)$ \\
& $0,199 * *$ & $-0,201 * * *$ & 0,077 & $-0,162 * * *$ \\
\multirow{2}{*}{ Lundi } & $(0,096)$ & $(0,064)$ & $(0,059)$ & $(0,054)$ \\
& $0,168^{*}$ & 0,085 & $0,139 * *$ & $-0,034$ \\
& $(0,096)$ & $(0,074)$ & $(0,069)$ & $(0,063)$ \\
\hline & $-4,737 * * *$ & $-6,590^{* * *}$ & $-5,037 * *$ & $-6,045^{* * *}$ \\
& $(0,908)$ & $(0,841)$ & $(0,894)$ & $(0,888)$
\end{tabular}




\begin{tabular}{|c|c|c|c|c|}
\hline Mardi & $\begin{array}{l}2,193^{*} \\
(1,236)\end{array}$ & $\begin{array}{l}-1,044 \\
(0,904)\end{array}$ & $\begin{array}{l}2,102^{*} \\
(1,221)\end{array}$ & $\begin{array}{l}-1,194 \\
(0,967)\end{array}$ \\
\hline Mercredi & $\begin{array}{l}1,401 \\
(1,085)\end{array}$ & $\begin{array}{c}-2,013 * * \\
(0,849)\end{array}$ & $\begin{array}{c}0,687 \\
(1,095)\end{array}$ & $\begin{array}{l}-1,644^{*} \\
(0,907)\end{array}$ \\
\hline Jeudi & $\begin{array}{l}-0,516 \\
(0,793)\end{array}$ & $\begin{array}{l}-1,042 \\
(0,670)\end{array}$ & $\begin{array}{l}-0,491 \\
(0,839)\end{array}$ & $\begin{array}{l}-1,092 \\
(0,693)\end{array}$ \\
\hline Vendredi & Ref & Ref & Ref & Ref \\
\hline Samedi & $\begin{array}{c}-2,856 * * * \\
(0,800)\end{array}$ & $\begin{array}{c}-4,027 * * * \\
(0,725)\end{array}$ & $\begin{array}{c}-3,612 * * * \\
(0,826)\end{array}$ & $\begin{array}{c}-3,619 * * * \\
(0,694)\end{array}$ \\
\hline $\begin{array}{l}\text { Coefficient de variation des prix } \\
\text { sur } 10 \text { jours }\end{array}$ & $\begin{array}{c}1,720 * * * \\
(0,416)\end{array}$ & $\begin{array}{c}1,977 * * * \\
(0,240)\end{array}$ & $\begin{array}{c}0,930 * * * \\
(0,308)\end{array}$ & $\begin{array}{c}1,292 * * * \\
(0,208)\end{array}$ \\
\hline Constante & $\begin{array}{c}5,559 * * * \\
(1,174)\end{array}$ & $\begin{array}{c}6,351 * * * \\
(0,845)\end{array}$ & $\begin{array}{c}7,005 * * * \\
(1,306)\end{array}$ & $\begin{array}{c}6,525^{* * * *} \\
(0,887)\end{array}$ \\
\hline Nombre d'observations & 732 & 732 & 732 & 732 \\
\hline
\end{tabular}

Lecture : Les colonnes contiennent les résultats des estimations par moindres carrés ordinaires. Les variables endogènes sont la fréquence de baisse (f-), et la fréquence de hausse de prix (f+) (en \%), par jour, les dimanches ont été exclus. Ces statistiques sont calculées pour l'ensemble des stations-service. L'évolution du prix de marché « Rotterdam » $\Delta p_{t-6, t}^{\text {Rotterdam }}$ est calculée sur une semaine précédant le jour t considéré. $\Delta p_{t-12, t-6}^{\text {Rotterdam }}, \Delta p_{t-18, t-12}^{\text {Rotterdam }}$ et $\Delta p_{t-24, t-18}^{\text {Rotterdam }}$ est l'évolution du prix Rotterdam retardé d'une, deux ou trois semaines. Le coefficient de variation des prix sur 2 semaines est l'écart-type du prix de marché sur les 10 jours ouvrables précédents sur le prix moyen constaté. Les estimations sont corrigées de l'autocorrélation à l'aide de la matrice de Newey-West. Niveau de significativité : *** $1 \%, * * 5 \%, * 10 \%$.

Source : Données individuelles quotidiennes issues du site internet www.prix-carburants.gouv.fr (stations service présentes plus de 500 jours dans la base de données, janvier 2007-juin 2009) et U.S. Energy Information Administration, calculs des auteurs.

Tableau A2 : Déterminants de la taille des changements de prix TTC

\begin{tabular}{lcccc}
\hline \hline & \multicolumn{2}{c}{ Diesel } & \multicolumn{2}{c}{ SP 95 } \\
& $d p+$ & $|d p-|$ & $d p+$ & $|d p-|$ \\
\hline \multirow{2}{*}{$\Delta p_{t-6, t}^{\text {Rotterdam }}$} & $-0,009 * * *$ & $0,007 * *$ & $-0,006^{* *}$ & 0,001 \\
$\Delta p_{t-12, t-6}^{\text {Rotterdam }}$ & $(0,003)$ & $(0,003)$ & $(0,003)$ & $(0,002)$ \\
$\Delta p_{t-18, t-12}^{\text {Rotterdam }}$ & $0,009^{*}$ & $-0,013^{* * *}$ & $-0,001$ & $-0,014 * * *$ \\
\multirow{2}{*}{$\Delta p_{t-24, t-18}^{\text {Rotterdam }}$} & $(0,005)$ & $(0,003)$ & $(0,002)$ & $(0,002)$ \\
& $-0,002$ & $-0,010^{* * *}$ & $-0,003$ & $-0,005^{* * *}$ \\
\multirow{2}{*}{ Lundi } & $(0,003)$ & $(0,003)$ & $(0,002)$ & $(0,002)$ \\
& 0,009 & 0,000 & 0,003 & $-0,001$ \\
Mardi & $(0,006)$ & $(0,003)$ & $(0,003)$ & $(0,002)$ \\
& $0,174 * * *$ & 0,007 & $0,217 * * *$ & 0,006 \\
Mercredi & $(0,035)$ & $(0,026)$ & $(0,032)$ & $(0,026)$ \\
& $-0,041$ & $-0,077 * * *$ & $-0,008$ & $-0,083 * * *$ \\
Jeudi & $(0,029)$ & $(0,027)$ & $(0,025)$ & $(0,025)$ \\
& $-0,029$ & $-0,082^{* * *}$ & $-0,006$ & $-0,095 * * *$ \\
Vendredi & $(0,024)$ & $(0,025)$ & $(0,022)$ & $(0,025)$ \\
& $-0,007$ & $-0,039$ & $-0,013$ & $-0,032$ \\
& $(0,021)$ & $(0,026)$ & $(0,018)$ & $(0,025)$ \\
& Ref & Ref & Ref & Ref
\end{tabular}




\begin{tabular}{lcccc} 
Samedi & $0,169 * * *$ & $0,042^{* *}$ & $0,174 * * *$ & 0,021 \\
& $(0,031)$ & $(0,020)$ & $(0,029)$ & $(0,020)$ \\
\hline \multirow{2}{*}{ Fréquence des changements } & $0,007 * * *$ & $0,012 * * *$ & $0,007 * * *$ & $0,010^{* * *}$ \\
Coefficient de variation des prix & $(0,002)$ & $(0,002)$ & $(0,002)$ & $(0,002)$ \\
sur 10 jours & $0,141^{* * *}$ & $0,098^{* * *}$ & $0,088^{* * *}$ & $0,070 * * *$ \\
\hline \multirow{2}{*}{ Constante } & $(0,014)$ & $(0,009)$ & $(0,010)$ & $(0,007)$ \\
\hline Nombre d'observations & $0,678^{* * *}$ & $0,760^{* * *}$ & $0,703 * * *$ & $0,776^{* * *}$ \\
& $(0,035)$ & $(0,031)$ & $(0,035)$ & $(0,033)$ \\
\hline
\end{tabular}

Lecture : Les colonnes contiennent les résultats des estimations par moindres carrés ordinaires. Les variables endogènes sont la taille moyenne des baisses (dp-), la taille moyenne des hausses de prix $(\mathrm{dp}+)(\mathrm{en} \%)$, par jour, les dimanches ont été exclus. Ces statistiques sont calculées pour l'ensemble des stations-service. L'évolution du prix de marché «Rotterdam» $\Delta p_{t-6, t}^{\text {Rotterdam }}$ est calculée sur une semaine précédant le jour t considéré. $\Delta p_{t-12, t-6}^{\text {Rotterdam }}, \Delta p_{t-18, t-12}^{\text {Rotterdam }}$ et $\Delta p_{t-24, t-18}^{\text {Rotterdam }}$ est l'évolution du prix Rotterdam retardé d'une, deux ou trois semaines. Le coefficient de variation des prix sur 2 semaines est l'écart-type du prix de marché sur les 10 jours ouvrables précédents sur le prix moyen constaté. Les estimations sont corrigées de l'autocorrélation à l'aide de la matrice de Newey-West. Niveau de significativité : *** 1\%, ** 5\%, *10\%.

Source : Données individuelles quotidiennes issues du site internet www.prix-carburants.gouv.fr (stations service présentes plus de 500 jours dans la base de données, janvier 2007-juin 2009) et U.S. Energy Information Administration, calculs des auteurs.

Tableaux A3 : Déterminants de la taille des changements de prix hors taxes par type de distributeurs

a) Tableau A3.1 : Supermarchés

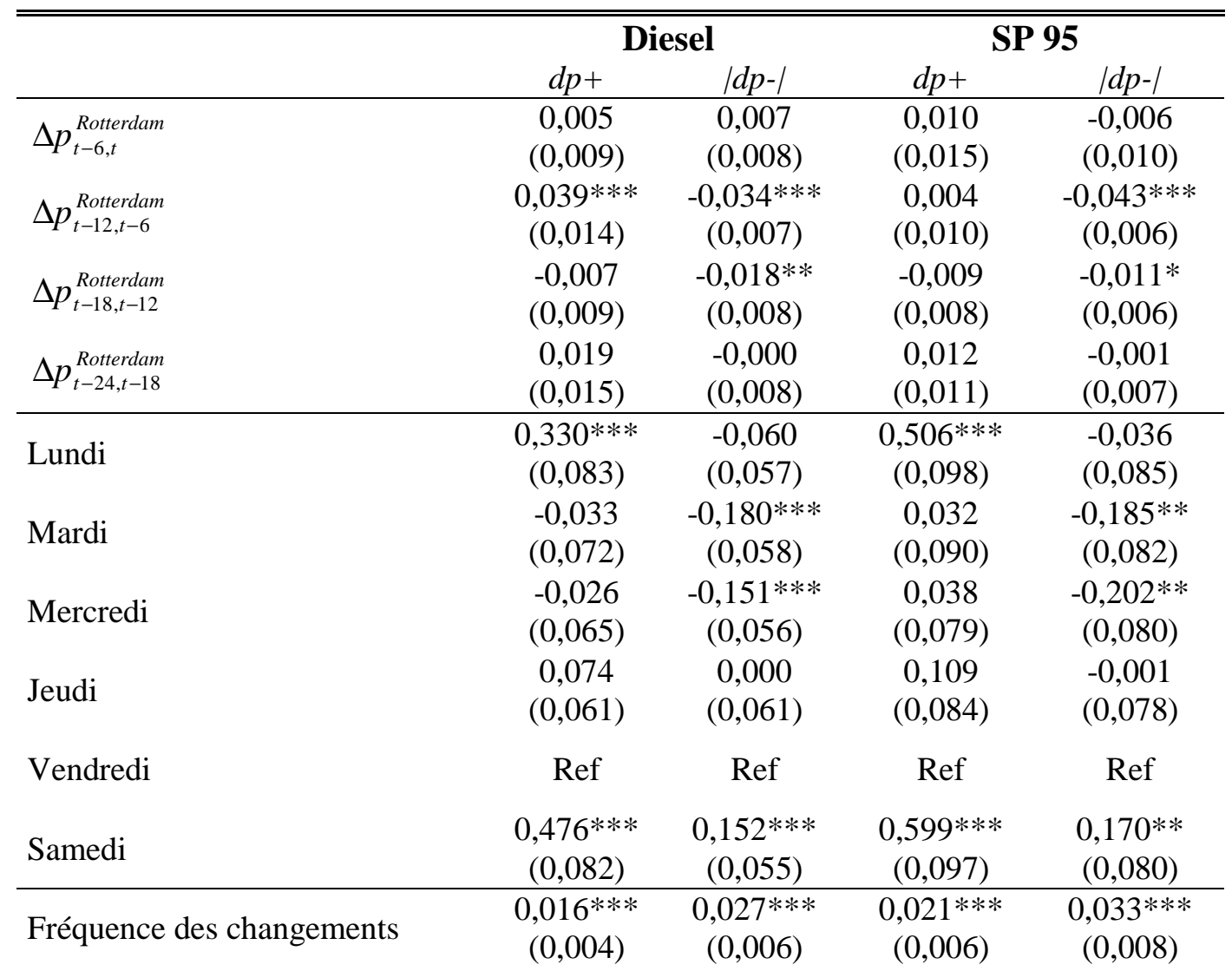




\begin{tabular}{lcccc}
$\begin{array}{l}\text { Coefficient de variation des prix } \\
\text { sur 10 jours }\end{array}$ & $\begin{array}{c}0,392 * * * \\
(0,041)\end{array}$ & $\begin{array}{c}0,273 * * * \\
(0,019)\end{array}$ & $\begin{array}{c}0,393 * * * \\
(0,042)\end{array}$ & $\begin{array}{c}0,298 * * * \\
(0,022)\end{array}$ \\
\hline \multirow{2}{*}{ Constante } & $0,854 * *$ & $1,056^{* * *}$ & $0,975 * * *$ & $1,213 * * *$ \\
& $(0,097)$ & $(0,093)$ & $(0,136)$ & $(0,127)$ \\
\hline Nombre d'observations & 731 & 732 & 732 & 731 \\
\hline
\end{tabular}

b) Tableau A3.2: Autres distributeurs

\begin{tabular}{|c|c|c|c|c|}
\hline & \multicolumn{2}{|c|}{ Diesel } & \multicolumn{2}{|c|}{ SP 95 } \\
\hline & $d p+$ & $|d p-|$ & $d p+$ & $|d p-|$ \\
\hline$\Delta p_{t-6, t}^{\text {Rotterdam }}$ & $\begin{array}{c}-0,023 * * * \\
(0,006)\end{array}$ & $\begin{array}{c}0,005 \\
(0,005)\end{array}$ & $\begin{array}{c}-0,036 * * * \\
(0,013)\end{array}$ & $\begin{array}{c}0,008 \\
(0,006)\end{array}$ \\
\hline$\Delta p_{t-12, t-6}^{\text {Rotterdam }}$ & $\begin{array}{c}0,002 \\
(0,007)\end{array}$ & $\begin{array}{c}-0,025^{* * *} * \\
(0,005)\end{array}$ & $\begin{array}{l}-0,011 \\
(0,007)\end{array}$ & $\begin{array}{c}-0,024 * * * \\
(0,005)\end{array}$ \\
\hline$\Delta p_{t-18, t-12}^{\text {Rotterdam }}$ & $\begin{array}{l}-0,002 \\
(0,007)\end{array}$ & $\begin{array}{c}-0,025^{* * *} \\
(0,006)\end{array}$ & $\begin{array}{l}-0,006 \\
(0,009)\end{array}$ & $\begin{array}{l}-0,007 \\
(0,006)\end{array}$ \\
\hline$\Delta p_{t-24, t-18}^{\text {Rotterdam }}$ & $\begin{array}{c}0,008 \\
(0,007) \\
\end{array}$ & $\begin{array}{l}-0,004 \\
(0,005) \\
\end{array}$ & $\begin{array}{c}0,000 \\
(0,009) \\
\end{array}$ & $\begin{array}{l}-0,002 \\
(0,004)\end{array}$ \\
\hline Lundi & $\begin{array}{c}0,190 * * * \\
(0,058)\end{array}$ & $\begin{array}{c}0,044 \\
(0,053)\end{array}$ & $\begin{array}{c}0,308 * * * \\
(0,090)\end{array}$ & $\begin{array}{c}0,089 \\
(0,075)\end{array}$ \\
\hline Mardi & $\begin{array}{l}-0,065 \\
(0,052)\end{array}$ & $\begin{array}{l}-0,074 \\
(0,045)\end{array}$ & $\begin{array}{c}0,027 \\
(0,077)\end{array}$ & $\begin{array}{c}-0,098 \\
(0,060)\end{array}$ \\
\hline Mercredi & $\begin{array}{l}-0,036 \\
(0,051)\end{array}$ & $\begin{array}{c}-0,139 * * * \\
(0,043)\end{array}$ & $\begin{array}{c}0,013 \\
(0,079)\end{array}$ & $\begin{array}{l}-0,111^{*} \\
(0,066)\end{array}$ \\
\hline Jeudi & $\begin{array}{l}-0,071 \\
(0,046)\end{array}$ & $\begin{array}{c}-0,107 * * \\
(0,048)\end{array}$ & $\begin{array}{l}-0,069 \\
(0,065)\end{array}$ & $\begin{array}{l}-0,089^{*} \\
(0,051)\end{array}$ \\
\hline Vendredi & Ref & Ref & Ref & Ref \\
\hline Samedi & $\begin{array}{c}0,190 * * \\
(0,074)\end{array}$ & $\begin{array}{l}-0,015 \\
(0,044)\end{array}$ & $\begin{array}{c}0,275 * * * \\
(0,097)\end{array}$ & $\begin{array}{c}0,009 \\
(0,066)\end{array}$ \\
\hline Fréquence des changements & $\begin{array}{l}-0,003 \\
(0,002)\end{array}$ & $\begin{array}{c}-0,007 * * \\
(0,003)\end{array}$ & $\begin{array}{c}0,001 \\
(0,004)\end{array}$ & $\begin{array}{l}-0,004 \\
(0,004)\end{array}$ \\
\hline $\begin{array}{l}\text { Coefficient de variation des prix } \\
\text { sur } 10 \text { jours }\end{array}$ & $\begin{array}{c}0,316 * * * \\
(0,024)\end{array}$ & $\begin{array}{c}0,209 * * * \\
(0,015)\end{array}$ & $\begin{array}{c}0,317 * * * \\
(0,032)\end{array}$ & $\begin{array}{c}0,257 * * * \\
(0,016)\end{array}$ \\
\hline Constante & $\begin{array}{c}1,375 * * * \\
(0,063)\end{array}$ & $\begin{array}{c}1,675 * * * \\
(0,051)\end{array}$ & $\begin{array}{c}1,499 * * * \\
(0,108)\end{array}$ & $\begin{array}{c}1,675 * * * \\
(0,079)\end{array}$ \\
\hline Nombre d'observations & 732 & 732 & 732 & 732 \\
\hline
\end{tabular}

Lecture : Les colonnes contiennent les résultats des estimations par moindres carrés ordinaires. Les variables endogènes sont la taille moyenne des baisses (dp-), la taille moyenne des hausses de prix $(\mathrm{dp}+)(\mathrm{en} \%)$, par jour, les dimanches ont été exclus. Ces statistiques sont calculées pour l'ensemble des stations-service. L'évolution du prix de marché "Rotterdam» $\Delta p_{t-6, t}^{\text {Rotterdam }}$ est calculée sur une semaine précédant le jour t considéré. $\Delta p_{t-12, t-6}^{\text {Rotterdam }}, \Delta p_{t-18, t-12}^{\text {Rotterdam }}$ et $\Delta p_{t-24, t-18}^{\text {Rotterdam }}$ est l'évolution du prix Rotterdam retardé d'une, deux ou trois semaines. Le coefficient de variation des prix sur 2 semaines est l'écart-type du prix de marché sur les 10 jours ouvrables précédents sur le prix moyen constaté. Les estimations sont corrigées de l'autocorrélation à l'aide de la matrice de Newey-West. Niveau de significativité : *** 1\%, ** 5\%,*10\%.

Source : Données individuelles quotidiennes issues du site internet www.prix-carburants.gouv.fr (stations service présentes plus de 500 jours dans la base de données, janvier 2007-juin 2009) et U.S. Energy Information Administration, calculs des auteurs. 


\title{
Résumé court
}

\section{L'ajustement microéconomique des prix des carburants en France}

\section{$\underline{\text { Résumé }}$}

En utilisant des millions de relevés individuels de prix entre 2007 et 2009, cette étude décrit plusieurs faits stylisés sur l'ajustement des prix des carburants en France : ils changent en moyenne une fois par semaine; ils sont plus souvent modifiés le mardi et le vendredi ; la concurrence accroît la fréquence des changements de prix; la distribution des tailles de changements de prix présente peu de changements inférieurs à $2 \%$; taille des changements de prix et durée des prix sont corrélées; l'inflation des prix des carburants est surtout déterminée par la part relative des stations-service augmentant ou baissant leur prix. Les modèles de coût d'ajustement incluant des rigidités informationnelles reproduisent le mieux ces faits stylisés.

Mots-clés : rigidité des prix, prix des carburants, inflation.

Codes JEL : E31, D43, L11.

\section{Gasoline price adjustment: some evidence from French individual data}

\begin{abstract}
$\underline{\text { Abstract }}$
Using millions of individual gasoline prices collected over the period 2007-2009, this study highlights several stylized facts on how gas stations adjust their prices in France: retail gasoline prices are changed once a week; price changes are more frequent on Tuesday and Friday; local competition has a positive impact on the frequency of price changes; price changes smaller than $2 \%$ are rare; average price duration and size of price changes are correlated; gasoline price inflation is mainly driven by variations in the frequency of price increases and decreases. Menu cost models including information rigidities reproduce most of these stylized facts.
\end{abstract}

Keywords: price stickiness, gasoline price, inflation.

JEL Codes: E31, D43, L11. 


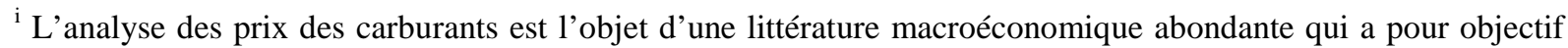
(souvent unique) de mesurer si la transmission des prix du pétrole aux prix du carburant est asymétrique (Audenis et al. (2002) et Lamotte et al. (2012) pour la France). Nous n'abordons pas cette question dans notre étude.

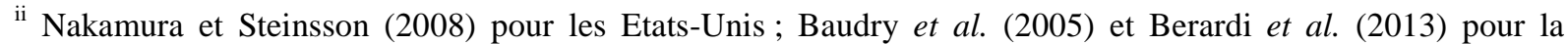
France ; Dhyne et al. (2006) pour la zone euro ; Klenow et Malin (2011) pour une synthèse des résultats de cette littérature.

iii Gautier et Le Saout (2012) proposent à l'aide d'estimations d'un modèle microéconométrique semi-structurel de coût d'ajustement d'analyser le lien entre la rigidité des prix des carburants et chocs économiques.

${ }^{\text {iv }}$ L'autorité française de la concurrence est en charge du site et de l'exactitude de l'information. Par exemple, entre janvier 2011 et juin 2011 environ 6000 stations-service ont été contrôlées et 72 procès-verbaux ont été dressés. De plus, 600000 consommateurs visitent régulièrement le site et peuvent déclarer (par courrier et téléphone) si le prix affiché sur le site est différent de celui constaté en station.

${ }^{\mathrm{v}}$ Les chiffres sont calculés à partir des comptes nationaux sectoriels publiés par l'Insee.

${ }^{\text {vi }}$ 60,69 euros par hectolitre pour le SP 95 et 42,84 euros par hectolitre pour le diesel, avec quelques faibles différences régionales. Cette taxe a été renommée en 2011 TICPE (Taxe Intérieure de Consommation sur les Produits Energétiques).

${ }^{\text {vii }}$ Calvet et Marical (2012) ou Bellec et al. (2012) soulignent que l'élasticité-prix est toutefois plus faible à court qu'à long terme

viii Ce ratio peut être rapproché de la part dans les coûts d'exploitation des stations-service.

${ }^{\text {ix }}$ Même s'il est plus difficile de justifier intuitivement l'existence de ces contrats, ces modèles sont les plus couramment utilisés par les modèles macroéconomiques car ils sont plus aisés à manipuler et reproduisent les dynamiques agrégées.

${ }^{\mathrm{x}}$ A moins de réaliser des enquêtes spécifiques au près des détaillants sur leur mode d'ajustement des prix comme celles menées par Blinder et al. (1998) aux Etats-Unis ou Fabiani et al. (2006) dans la zone euro.

${ }^{x i}$ Voir par exemple Baudry et al. (2005) ou Gautier (2008) pour les définitions et les notations utilisées.

${ }^{\text {xii }}$ Les fonctions de hasard présentent des profils relativement semblables pour les supermarchés ou les autres stations-service.

xiii Même si ce coût devient plus faible grâce aux services internet dédiés aux comparaisons de prix des carburants par exemple.

xiv L'effet des supermarchés est évalué comme la différence de la somme des coefficients associés aux indicatrices de marques de supermarchés et la même somme pour les autres types de stations, l'écart-type étant obtenu par la delta-méthode.

${ }^{\mathrm{xv}}$ Nos résultats sont robustes au choix du rayon, nous avons testé 2,5 et $10 \mathrm{~km}$

${ }^{x v i}$ Des régressions ont aussi été menées en considérant séparément les stations de supermarchés et les autres stations. Les résultats obtenus sont proches.

${ }^{x v i i}$ Asplund et al. (2000) sur des données de carburants suédois obtiennent qu'aucun changement de prix n'est inférieur à $2 \%$.

xviii Gautier et Le Saout (2012) obtiennent par exemple que pour le diesel, la part de la matière première dans le coût marginal est de l'ordre de 0,8 pour les supermarchés alors qu'il n'est que de 0,7 pour les autres stations.

${ }^{\text {xix }}$ Comparé au prix du Brent, le prix du produit fini coté à Rotterdam présente l'intérêt d'inclure des coûts de raffinage et est donc plus « proche » du produit étudié (Meyler, 2010).

${ }^{\mathrm{xx}}$ Des résultats identiques sont obtenus sur les prix TTC.

${ }^{x x i}$ Les inflations sont ici calculées par semaine en sommant les inflations quotidiennes pour rendre la représentation plus lisible.

xxii Nous avons aussi mené des régressions séparées pour les supermarchés et les autres stations (Tableau A1 en annexe) et les résultats sont proches pour les deux types de stations même si l'ajustement semble plus rapide pour les supermarchés.

${ }^{\text {xxiii }}$ Pour ce calcul, on utilise la formule $\pi_{t}^{*} \mid f=f_{t}^{+} \times \overline{d p^{+}}+f_{t}^{-} \times \overline{d p^{-}}$, on simule jour après jour suivant le choc l'effet sur les fréquences de hausses et de baisses en utilisant les résultats du tableau 7 , on reconstruit ainsi une inflation incorporant le choc et on la compare à une inflation sans choc 3 semaines après la hausse de $1 \%$ du prix Rotterdam. Par construction, il n'y a pas ici d'asymétrie de réaction entre hausses et baisses du prix Rotterdam.

xxiv Asplund et al. (2000) sur des données suédoises obtiennent des résultats similaires, dans leur cas, c'est le mardi et le mercredi où les changements de prix sont les plus fréquents alors qu'entre le samedi et le lundi il y a très peu de changements de prix. 
${ }^{\mathrm{xxv}}$ Nous avons également estimé le modèle en utilisant les prix TTC et les résultats sont similaires, les effets des différentes variables sur les prix TTC étant amortis par la présence des taxes (Tableau A2 en annexe).

${ }^{x x v i}$ Les résultats sont similaires en considérant séparément les supermarchés ou les autres stations-services (Tableau A3 en annexe). 